\title{
Low-Complexity MU-MIMO Nonlinear Precoding Using Degree-2 Sparse Vector Perturbation
}

\author{
Yi Ma, Senior Member, IEEE, Abderraouf Yamani, Na Yi, Member, IEEE, \\ and Rahim Tafazolli, Senior Member, IEEE
}

Invited Paper

\begin{abstract}
Multiuser multiple-input multiple-output (MUMIMO) nonlinear precoding techniques face the problem of poor computational scalability to the size of the network. In this paper, the fundamental problem of MU-MIMO scalability is tackled through a novel signal-processing approach, which is called degree-2 vector perturbation (D2VP). Unlike the conventional VP approaches that aim at minimizing the transmit-to-receive energy ratio through searching over an $N$-dimensional Euclidean space, D2VP shares the same target through an iterative-optimization procedure. Each iteration performs vector perturbation over two optimally selected subspaces. By this means, the computational complexity is managed to be in the cubic order of the size of $\mathrm{MU}$ MIMO, and this mainly comes from the inverse of the channe matrix. In terms of the performance, it is shown that D2VP offers comparable bit-error-rate to the sphere encoding approach for the case of small MU-MIMO. For the case of medium and large MU-MIMO when the sphere encoding does not apply due to unimplementable complexity, D2VP outperforms the latticereduction VP by around $5-10 \mathbf{d B}$ in $\mathbf{E b} / \mathbf{N o}$ and $10-50 \mathbf{d B}$ in normalized computational complexity.
\end{abstract}

Index Terms-Low complexity, multiuser multiple-input multiple-output (MU-MIMO), nonlinear precoding, vector perturbation.

\section{INTRODUCTION}

$\mathbf{N}$ EXT generation of broadband mobile internet (namely $5 \mathrm{G})$ is expected to support several orders of magnitude in capacity compared with that in $4 \mathrm{G}$ and its evolutions. There are several ways of achieving such huge capacity through densification of cells, massive multiple-input multiple-output (MIMO) and new extended bandwidth and their combination [1]. This paper presents a novel multiuser (MU) MIMO nonlinear precoding (NLP) approach for the downlink of MU-MIMO networks, of which the computational complexity scales linearly with the size of the MIMO networks, and the performance can be better than the state-of-the-art by $10 \mathrm{~dB}$ or more in $\mathrm{Eb} / \mathrm{No}$.

\section{A. Background and Motivation}

In the area of multiuser information theory, the downlink of MU-MIMO networks can be modelled as MIMO Gaussian

Manuscript received August 10, 2015; revised October 15, 2015. This work was supported in part by the UK 5G Innovation Centre, and in part by European Commission under the framework of the FP7 project ICT619555 RESCUE (Links-on-the-fly Technology for Robust, Efficient and Smart Communication in Unpredictable Environment).

The authors are with the Institute for Communication Systems (ICS), University of Surrey, Guildford, UK, GU2 7XH. (e-mail: y.ma@surrey.ac.uk, a.yamani@surrey.ac.uk,n.yi@surrey.ac.uk,r.tafazolli@surrey.ac.uk).

This work has been filed in an UK patent with application no. GB1420944.9 and international patent with application no. PCT74933. broadcast channel. Theoretically, the sum-rate capacity of such a channel grows linearly with the number of spatial-domain DoF [2], [3], and it can be achieved through multi-antenna dirty-paper coding (DPC) [3], [4]. However, the practical implementation of multi-antenna DPC faces great challenges of computational scalability to the size of the MIMO network; and today only small-scale MU-MIMO (up to $8 \times 8$ ) with linear precoding is adopted in $4 \mathrm{G}$ standards.

In the last decade, a number of remarkable contributions have been reported in the scope of multi-antenna DPC, which include nested lattice [5], [6], trellis precoding [7], V-BLAST precoding [8] and vector perturbation (VP) [9]. It has been shown that the VP technique can achieve near-optimum performance at all signal-to-noise ratios (SNRs) [10]. Nevertheless, the VP technique also faces big problems of the computational scalability. Specifically, the optimum VP technique aims at solving an integer least-square (ILS) problem, which theoretically requires exhaustive search over an infinite set of integers in the $N$-dimensional Euclidean space. Such an approach costs infinite number of arithmetic operations, and thus it is not possible to implement. The sphere encoding (SE) VP approach proposed in [10] successfully avoids the problem of infinite searching by conducting tree searching over a finite set of integers. However, the computational complexity of SE-VP still scales exponentially with the size of the network.

One of sustainable approaches that can dramatically reduce the computational complexity is the lattice reduction (LR), which has been widely investigated for both the uplink MIMO detection [11] and the downlink MIMO nonlinear precoding (NLP) [12]. It has been shown that the LR-VP approach is a sub-optimum NLP at the cost of computational complexity between $\mathcal{O}\left(N^{4}\right)$ and $\mathcal{O}\left(N^{5}\right)$. Such a large reduction in computational complexity is certainly impressive. However, the complexity is still too high for the LR-VP approach to be implemented using the current digital-signal-processor (DSP) technology. It is possible to further trade off the performance for lower complexity through for instance the V-BLAST approach [8], [10]. However, the V-BLAST approach still costs expensive computational complexity, which is in the order of $\mathcal{O}\left(N^{4}\right)$. Moreover, the performance of V-BLAST is only 2-3 $\mathrm{dB}$ better than that of linear zero-forcing $(\mathrm{ZF})$ precoding.

Certainly, one can find more MU-MIMO NLP approaches in the literature (e.g. [13], [14]). Most of existing approaches were looking for a good tradeoff between the performance and complexity. The question is: is it possible to find a precoding technique that can show excellence in both the performance 
and complexity? If the answer is "yes", then such a technique can bring MU-MIMO NLP much closer to fruition.

\section{B. Contribution}

Motivated by the above question, a novel MU-MIMO NLP approach, named degree-2 vector perturbation (D2VP), is proposed in this paper. It will be shown that the D2VP approach outperforms the LR-VP approach by around $5-10$ $\mathrm{dB}$ in SNR (subject to the size of MU-MIMO networks), and it manages the computational complexity in the order of $\mathcal{O}\left(N^{3}\right)$, which mainly comes from the inverse of the MIMO channel matrix. Therefore, the D2VP approach offers competitive computational complexity in comparison to the linear ZF approach (see [15], [16]) with more than $10 \mathrm{~dB}$ performance improvement in SNR.

The basic idea of D2VP comes from an important phenomenon: for the VP technique aiming to minimize the transmit-to-receive energy ratio, the majority of the contribution comes from a small portion of the subspaces in the $N$-dimensional Euclidean space ${ }^{1}$. This means that the perturbation vector is sparse in nature. With this interesting phenomenon in mind, the VP optimization process does not need to search the entire Euclidean space for the global optimum point. Instead, it can break down the optimization process into several iterations, with each performing local optimization based on two optimally selected subspaces. By this means, given a finite set of integers with the size $K$, the complexity paid for exhaustive search in the two selected subspaces is $\mathcal{O}\left(K^{2}\right)$. In fact, the exhaustive search is not needed in the D2VP optimization process. In Section III, it will be shown that D2VP forms a simple convex optimization problem, of which the local optimum point can be found in a closed form. This immediately reduces the optimization complexity from the square order to linear. In addition to the new concept, other major contributions of this paper include:

- Determine the subspaces of interest in the iterative process of D2VP. To this end, an optimum D2VP approach is developed through exhaustive search over all possible combinations of the subspaces. This approach ensures the best combination of subspaces with the complexity of $\mathcal{O}(N(N-1))$. In order to reduce the computational complexity, a complexity-reduced (CR) D2VP approach is proposed by selecting the subspaces which minimize the impact of the largest singular values of the channel inverse matrix. Performance comparison between the two proposed approaches will be conducted in Section V.

- The convergence behaviour of the D2VP iterative process is carefully investigated. It will be shown that, for the small and medium size of MU-MIMO (e.g. $N \leq 64$ ), the D2VP iterative process quickly converges to a local optimum point within 1-3 iterations. For the large size of MU-MIMO (e.g. $N=128$ or above), the performance can be further improved through one or two more iterations. However, the improvement is not comparable with the first three iterations.

\footnotetext{
${ }^{1}$ This interesting phenomenon was first discovered in [10], and similar results will be also discussed in Section V of this paper.
}

- Generally, the perturbation vector can be either complex or real. In Section V, it will be shown that the real version offers comparable performance to the complex version when the size of MU-MIMO is sufficiently large (e.g. $N \geq 64$ ). Therefore, the real version can be a better approach for the case of large MU-MIMO due to its relatively low computational complexity.

- In fully-loaded MU-MIMO systems, most of VP-based NLP approaches do not get their performance improved when the size of MU-MIMO increases. Our computer simulations show that it is not the case for the D2VP approach. When the size of MU-MIMO is small (e.g. $N \leq 8$ ), the optimum D2VP approach has its performance improved with the increase of the MU-MIMO size. It means that the optimum D2VP can enjoy the spatialdomain diversity gain in the case of small MU-MIMO.

- In addition to the D2VP approach, we have also experimentally examined an extended approach, which is called degree-3 vector perturbation (D3VP). Although D3VP largely increases the computational complexity, our computer simulations show that it outperforms D2VP by up to $2 \mathrm{~dB}$ in SNR for the case of small MU-MIMO. This result encourages us to investigate the best performancecomplexity tradeoff of the sparse vector perturbation, which could be a piece of interesting future work.

The rest of this paper is organized as follows. Section II is the preliminary section, which includes the system model of MU-MIMO, basic assumptions, concept of vector perturbation, as well as the problem formulation. The basic concept of D2VP and the algorithm optimization are presented in Section III. The RC-D2VP approach is presented in Section IV. Section $\mathrm{V}$ provides the simulation results and performance evaluation. The conclusion is drawn in Section VI.

\section{PREliminary}

\section{A. Vector Perturbation and Optimization}

The original work of VP is built upon a discrete-time equivalent baseband model, which describes the link-level of a wireless system including an access point with $M$ transmit antennas and $N$ individual users. Each user has one receive antenna (see [9]). The observed signal at the $n^{\text {th }}$ user is

$$
\mathrm{y}_{n}=\mathbf{h}_{n}^{T} \mathbf{x}+\mathrm{v}_{n}, n \in[1, N],
$$

where the superscript $[\cdot]^{T}$ is the matrix transpose, $\mathbf{h}_{n}=$ $\left[\mathrm{h}_{n, 1}, \ldots, \mathrm{h}_{n, M}\right]^{T}$ the channel vector with its $m^{\text {th }}$ element, $\mathrm{h}_{n, m}$, denoting the channel coefficient between the $m^{\text {th }}$ transmit antenna and the $n^{\text {th }}$ receive antenna, $\mathrm{x}=\left[\mathrm{x}_{1}, \ldots, \mathrm{x}_{M}\right]^{T}$ the transmitted signal block with its $m^{\text {th }}$ element, $\mathrm{x}_{m}$, denoting the signal sent by the $m^{\text {th }}$ transmit antenna, and $\mathrm{v}_{n}$ the standard complex Gaussian noise at the $n^{\text {th }}$ user.

It is assumed that the access point has the global channel knowledge of $\mathrm{h}_{n, m}, n \in[1, N], m \in[1, M]$, which is represented by an $N \times M$ channel matrix $\mathbf{H}$. The function of VP is relating the transmitted signal $\mathrm{x}$ to the information symbols $\mathrm{s}_{n}$

$$
\mathbf{x}=\mathbf{H}^{\dagger}(\mathbf{s}+\alpha \boldsymbol{\omega})
$$


where the superscript $[\cdot]^{\dagger}$ is the matrix pseudo inverse, $\mathbf{s}=$ $\left[\mathrm{s}_{1}, \ldots, \mathrm{s}_{\mathrm{N}}\right]^{T}, \boldsymbol{\omega}=\left[\omega_{1}, \ldots, \omega_{N}\right]^{T}$ with $\omega_{n}$ to be integer, and $\alpha$ a scaling factor which is optimally configured with respect to the modulation format of $\mathbf{s}$ (see [10]). Plugging (2) into (1) yields

$$
\mathrm{y}_{n}=\mathrm{s}_{n}+\alpha \omega_{n}+\mathrm{v}_{n} .
$$

The user knows the modulation format of $\mathrm{s}_{n}$ and thus also the scaling factor $\alpha$ through a look-up table. A modulo receiver can be employed to remove the term $\alpha \omega_{n}$ from (3) by means of

$$
\mathrm{z}_{n}=\mathrm{y}_{n}-\alpha\left\lfloor\frac{\mathrm{y}_{n}+\alpha(1+j) / 2}{\alpha}\right\rfloor,
$$

where $\lfloor\cdot\rfloor$ denotes the integer floor. Then, a signal detector can be applied on $\mathrm{z}_{n}$ to reconstruct $\mathrm{s}_{n}$, and the transmit-receive procedure completes.

The aim of VP is to find the optimum configuration of $\boldsymbol{\omega}$ (denoted by $\omega^{*}$ ) with the following objective function to be minimized

$$
\boldsymbol{\omega}^{*}=\underset{\boldsymbol{\omega}}{\operatorname{argmin}} \frac{\left\|\mathbf{H}^{\dagger}(\mathbf{s}+\alpha \boldsymbol{\omega})\right\|^{2}}{E_{\mathrm{s}}},
$$

where $E_{\mathrm{S}}$ is the received signal energy, and $\|\cdot\|$ the Frobenius norm. By this means, the optimum configuration $\omega^{*}$ maximizes the transmit-energy efficiency.

When $E_{\mathrm{s}}$ is forced to be constant, (5) is equivalent to

$$
\boldsymbol{\omega}^{*}=\underset{\boldsymbol{\omega}}{\operatorname{argmin}}\left\|\mathbf{H}^{\dagger}(\mathbf{s}+\alpha \boldsymbol{\omega})\right\|^{2}
$$

Defining $\overline{\mathbf{s}} \triangleq \mathbf{H}^{\dagger} \mathbf{s}$ and re-defining $\boldsymbol{\omega} \triangleq-\boldsymbol{\omega}$, (6) immediately becomes

$$
\boldsymbol{\omega}^{*}=\underset{\boldsymbol{\omega}}{\operatorname{argmin}}\left\|\overline{\mathbf{s}}-\alpha \mathbf{H}^{\dagger} \boldsymbol{\omega}\right\|^{2} .
$$

Then, the objective function of VP defines an integer leastsquare (ILS) problem. It is worthwhile to note that the difference $\left(\overline{\mathbf{s}}-\alpha \mathbf{H}^{\dagger} \boldsymbol{\omega}\right)$ comes from the integer property of $\boldsymbol{\omega}$ instead of the thermal noise. Therefore, the ILS problem in (7) differs from the ILS problem in the MU-MIMO detection (e.g. [17]) mainly in three folds:

- The difference $\left(\overline{\mathbf{s}}-\alpha \mathbf{H}^{\dagger} \boldsymbol{\omega}\right)$ is not a Gaussian process.

- The minimum of $\left\|\overline{\mathbf{s}}-\alpha \mathbf{H}^{\dagger} \boldsymbol{\omega}\right\|^{2}$ is not zero or $\mathbb{E}(\overline{\mathbf{s}}-$ $\left.\alpha \mathbf{H}^{\dagger} \boldsymbol{\omega}\right) \neq 0$, where $\mathbb{E}(\cdot)$ denotes the expectation.

- Theoretically, elements of $\boldsymbol{\omega}$ are selected from an infinite set of integers.

Hence, the ILS problem in (7) does not lead to a maximumlikelihood solution. Moreover, the linear minimum meansquare error approach widely used for the MU-MIMO detection is not applicable to (7). On the other hand, the principle of sphere decoding can still be employed to solve (7) as long as $\boldsymbol{\omega}$ is restricted to a finite set of integers (this is known as the SE-VP approach); and the lattice-reduction approach can also be employed to regularize the channel inverse matrix $\mathbf{H}^{\dagger}$. Nevertheless, as we have already discussed in Section I, none of existing approaches is saturated in terms of the performance-complexity tradeoff, and thus the D2VP approach is motivated.

\section{B. Application Scenarios and Fundamental Assumptions}

The application scenarios of the VP-NLP technique have already been well interpreted in [9]. Despite, we find it still important to elaborate several key technical issues with some new ingredients.

1) About the channel matrix $\mathbf{H}$ : We believe that most of the interesting questions would come to the channel matrix $\mathbf{H}$ regarding its availability at the transmitter side, its dimension and regularity.

Section II-A has shown that the VP technique requires the knowledge of $\mathbf{H}$ to be made available at the transmitter side for the sake of channel inverse and regularization. This requirement is indeed demanding in today's wireless networks. However, it is not unrealistic as far as low-mobility timedivision duplexing (TDD) networks are concerned. In Europe, wireless researchers have already been working towards the channel reciprocity in MIMO/TDD test networks (e.g. [18]), which if successful enables the use of uplink channel estimates for the downlink MU-MIMO precoding. In the pessimistic cases when the full knowledge of $\mathbf{H}$ is not available at the transmitter side, there have been already remarkable progress on the MU-MIMO precoding techniques with imperfect CSIT, delayed CSIT, or limited feedback (e.g. [19]-[21]), for which the technique with full CSIT provides the upper bound of the data rate.

Regarding the dimension and regularity of $\mathbf{H}$, we found the discussion in [9] already quite comprehensive. Here, we stress that the number of spatial-domain DoF equals to the rank of $\mathbf{H}$, which puts an upper limit onto the number of orthogonal data-streams. Without loss of generality, we therefore can assume $M \geq N$ and $N=\operatorname{Rank}(\mathbf{H})$. It is worthwhile to note that future wireless networks will be super dense in nature, and very demanding to the highly spectral efficiency. To this end, the number of spatial-domain DoF should be as large as possible, and thus the spatial domain is very likely to be fully loaded. In this case, the linear MU-MIMO precoding techniques are far away from the optimum [22]. Therefore, in the rest of the paper, we consider the case $M=N$ for its critical position in future super dense networks.

2) User equality in date rate: In the original VP problem, it is assumed that all the users (receivers) have the same data rate (modulation). We also recognize this as a practical assumption. For instance in UMTS or LTE-A networks, the data rate is often region specific. The data regions are classified according to the large-scale path loss between the transmitter and receivers [23], [24]. In this case, users located in the same data region can be scheduled with the VP-based spatial-domain multipleaccess (SDMA), and those located in different data regions can be scheduled on different time or frequency resources.

3) Synchronization issues: We recognize synchronization as one of critical issues in the area of MU-MIMO processing. The issue of timing synchronization can be relatively easy to solve by employing the time-domain guard interval or cyclic prefix. However, the frequency synchronization is indeed a big concern for the practical implementation of MU-MIMO systems. Nevertheless, there have been already a lot of ongoing research activities in the scopes of synchronization and waveform design (e.g. [25]), and thus in this paper we assume 
the perfect case of synchronization so that our technical presentation can be focused on the immediate problem of interest.

\section{Degree-2 Sparse Vector Perturbation}

\section{A. Concept and Rationale}

Definition 1: The sparsity of perturbation vector refers to the phenomenon: every element of $\boldsymbol{\omega}$ has a large probability to be zero after the vector perturbation. Equivalently, when the size of $\boldsymbol{\omega}$ is large, most of the elements in $\boldsymbol{\omega}$ are zero.

According to the simulation results in [14], the probability for an element of $\boldsymbol{\omega}$ to be zero is around $80 \%$ or more. This phenomenon has been confirmed through our computer simulations (see Section V).

Definition 2: D2VP is a low-complexity vector perturbation technique, which takes advantage of the sparsity of perturbation vector in the matrix-regularization procedure. Similar to the original VP technique, the objective of D2VP is also to handle the ILS problem (7). Instead of manipulating all the elements of the perturbation vector $\boldsymbol{\omega}$, the idea of D2VP is to break down the matrix-regularization process into several iterations, each performing the matrix-regularization based on two appropriately selected elements of $\boldsymbol{\omega}$.

Let us take the $i^{\text {th }}$ iteration as an example to elaborate the basic concept of D2VP. Note that, for each iteration, the terms $\overline{\mathbf{s}}$ and $\boldsymbol{\omega}^{*}$ will be updated, and thus in the following expressions they are labelled with the index $(i)$. Then, the objective function of the $i^{\text {th }}$ iteration is

$$
\boldsymbol{\omega}^{*}(i)=\underset{\boldsymbol{\omega}}{\operatorname{argmin}}\left\|\overline{\mathbf{s}}(i-1)-\alpha \mathbf{H}^{\dagger} \boldsymbol{\omega}\right\|^{2},
$$

where $\overline{\mathbf{s}}(0)=\overline{\mathbf{s}}$ and

$$
\overline{\mathbf{s}}(i)=\overline{\mathbf{s}}(i-1)-\alpha \mathbf{H}^{\dagger} \boldsymbol{\omega}^{*}(i) .
$$

As long as the iterative process terminates, we can obtain

$$
\mathbf{x}=\overline{\mathbf{s}}(I) \text { and } \boldsymbol{\omega}^{*}=\sum_{i=1}^{I} \boldsymbol{\omega}^{*}(i),
$$

where $I$ is the number of iterations.

1) The objective function of D2VP: In order to clarify the concept of D2VP, here assume that the perturbation vector $\omega$ is real (see Section III-C for the complex version). Suppose there are two elements of $\boldsymbol{\omega}$ ( say $\omega_{n_{1}}, \omega_{n_{2}}, n_{1}, n_{2} \in[1, N], n_{1} \neq n_{2}$ ), which has been selected for the D2VP processing. Then, the other $(N-2)$ elements, which were not selected, are set to zero. By defining $\boldsymbol{\omega}_{2} \triangleq\left[\omega_{n_{1}}, \omega_{n_{2}}\right]^{T}$ and $\widetilde{\mathbf{H}}$ an $N \times 2$ matrix formed by the $n_{1}{ }^{\text {th }}$ and $n_{2}{ }^{\text {th }}$ column of $\mathbf{H}^{\dagger}$, the objective function (8) immediately becomes

$$
\boldsymbol{\omega}_{2}^{*}(i)=\underset{\boldsymbol{\omega}_{2}}{\operatorname{argmin}}\left\|\overline{\mathbf{s}}(i-1)-\alpha \widetilde{\mathbf{H}} \boldsymbol{\omega}_{2}\right\|^{2},
$$

and then $\boldsymbol{\omega}^{*}(i)$ is formed through zero padding of $\boldsymbol{\omega}_{2}^{*}(i)$.

We can see that the VP technique based on (11) is in line with Definition 2. Basically, the objective function (11) can be solved through several approaches, which include the exhaustive search, sphere encoding, and V-BLAST. However, those approaches require $\omega_{2}$ to be restricted to a finite set of integers, and thus they would suffer optimality loss. Moreover, the complexity paid for searching over the finite set is in the square order. Note that the optimization procedure of (11) requires to visit all possible states of $\left(n_{1}, n_{2}\right)$, which also costs square-order complexity. Then, the overall cost of computational complexity is in the order of four, which is certainly too expensive. In Section III, we will demonstrate an optimum D2VP approach, with which the optimum point of (11) can be found in a closed form.

2) Iterative process and convergence: In general, D2VP is a sub-optimum VP approach due to the reduced number of subspaces (or equivalently the reduced number of nonzero elements in $\boldsymbol{\omega}$ ) involved in the optimization procedure. Nevertheless, the optimality of D2VP can be improved through the iterative process described by (8)-(10).

Consider the outcome of the $i^{\text {th }}$ iteration, i.e., $\boldsymbol{\omega}^{*}(i)$ and $\overline{\mathbf{s}}(i)$. The vector $\boldsymbol{\omega}^{*}(i)$ has two possible states: 1) $\boldsymbol{\omega}^{*}(i)=\mathbf{0}$, or 2) $\boldsymbol{\omega}^{*}(i) \neq \mathbf{0}$. For the state of $\boldsymbol{\omega}^{*}(i)=\mathbf{0}$, (9) shows that $\overline{\mathbf{s}}(i)=\overline{\mathbf{s}}(i-1)$, with which more iterations would not further improve the performance, and thus the iterative process terminates. For the state of $\boldsymbol{\omega}^{*}(i) \neq \mathbf{0}$, the objective function (8) assures $\|\overline{\mathbf{s}}(i)\|<\|\overline{\mathbf{s}}(i-1)\|$, which means that the performance of D2VP has been further improved. Note that each iteration aims at reaching a local optimum based on the previous outcome $\overline{\mathbf{s}}(i-1)$, therefore the iterative process functions as the neighbourhood search [26], which will quickly converge to a local optimum solution.

It is also worthwhile to note that, after $I$ iterations, the number of non-zero elements in $\boldsymbol{\omega}$ can be up to $2 I$ instead of 2. This is because each iteration has a different version of $\overline{\mathbf{s}}(i-1)$, with which the optimum state of $\left(n_{1}, n_{2}\right)$ is different from the previous one.

3) Optimality analysis for D2VP: We start from the original VP problem formulated in (2) and (7). The Frobenius norm of $\mathbf{x}$ is given by

$$
\|\mathbf{x}\|^{2}=\sum_{l=1}^{N} \underbrace{\left|\overline{\mathrm{s}}_{l}-\alpha \sum_{n=1}^{N} \tilde{\mathrm{h}}_{l, n} \omega_{n}\right|^{2}}_{\triangleq \beta_{l}}
$$

where $\overline{\mathrm{s}}_{l}$ denotes the $l^{\text {th }}$ element of $\overline{\mathrm{s}}$, and $\tilde{\mathrm{h}}_{l, n}$ the $(l, n)^{\text {th }}$ element of $\mathbf{H}^{\dagger}$. Now, the aim is to find a set of $\left\{\beta_{1}, \ldots, \beta_{N}\right\}$, which minimizes $\|\mathbf{x}\|^{2}$.

Given two indices $n_{1}, n_{2} \in[1, N]$, (12) can be rewritten into

$$
\beta_{l}=\left|\overline{\mathrm{s}}_{l}-\alpha \sum_{\substack{n=n_{1} \\ n=n_{2}}} \tilde{\mathrm{h}}_{l, n} \omega_{n}-\alpha \sum_{\substack{n \neq n_{1} \\ n \neq n_{2}}} \tilde{\mathrm{h}}_{l, n} \omega_{n}\right|^{2} .
$$

Then, we form the relationship

$$
\sum_{\substack{n \neq n_{1} \\ n \neq n_{2}}} \tilde{\mathrm{h}}_{l, n} \omega_{n}=\sum_{\substack{n=n_{1} \\ n=n_{2}}} \tilde{\mathrm{h}}_{l, n} \omega_{n}^{\prime}+\varepsilon_{l}
$$

where $\omega_{n}^{\prime}$ is the corresponding integer, and $\varepsilon_{l}$ the difference between two sums in (14), of which the amplitude can be minimized by means of configuring $\omega_{n}^{\prime}$. 
Plugging (14) into (13) leads to

$$
\beta_{l}=\left|\overline{\mathrm{s}}_{l}-\alpha \sum_{\substack{n \neq n_{1} \\ n \neq n_{2}}} \tilde{\mathrm{h}}_{l, n}\left(\omega_{n}+\omega_{n}^{\prime}\right)-\alpha \varepsilon_{l}\right|^{2} .
$$

Since $\left(\omega_{n}+\omega_{n}^{\prime}\right)$ is still an integer, we can omit the term $\omega_{n}^{\prime}$ and simplify (15) into

$$
\beta_{l}=\left|\overline{\mathrm{s}}_{l}-\alpha \sum_{\substack{n \neq n_{1} \\ n \neq n_{2}}} \tilde{\mathrm{h}}_{l, n} \omega_{n}-\alpha \varepsilon_{l}\right|^{2} .
$$

The reason of having $\varepsilon_{l}$ in (14)-(16) is because of the constraint that $\omega_{l}$ is integer. We adopt the same assumption as the one used in [9], [10]: $\tilde{\mathrm{h}}_{l, n, \forall l, n}$, is independently generated according to the standard complex Gaussian distribution. Then, the impact of the integer constraint decreases when $N$ becomes large; and we have $\varepsilon_{l} \rightarrow 0$ when $N \rightarrow \infty$. As a consequence, in the procedure of computing $\beta_{l}$, D2VP suffers optimality loss in the small size of MU-MIMO, and such a loss approximates to zero in the large size of MU-MIMO.

The other issue causing the optimality loss is: $\beta_{l, \forall l \in[1, N] \text {, }}$ must be jointly optimized in order to minimize $\|\mathbf{x}\|^{2}$. For instance given two indices $l_{1}, l_{2} \in[1, N]$, when we find a candidate of $\boldsymbol{\omega}\left(\right.$ or $\left.\omega_{2}\right)$ that reduces $\beta_{l_{1}}$, it might increase $\beta_{l_{2}}$ at the same time. As a consequence, such a candidate might not minimise $\|\mathbf{x}\|^{2}$. In this aspect, the D2VP approach has less spatial-domain DoF to exploit when comparing with the optimum VP approach. On the other hand, the iterative process facilitates D2VP with increased number of DoF, and it can lead D2VP to a better sub-optimum solution. The optimality of D2VP will be further examined through computer simulations in Section V.

\section{B. The Optimum D2VP Approach}

In this section, it will be shown that the objective function (11) has a closed-form solution, for which the optimum decision $\omega_{2}^{*}$ is formed with low computational complexity.

In order to demonstrate a clear pathway to the optimum solution of D2VP, our derivation will start from the continuous version of $\boldsymbol{\omega}_{2}$, which is defined by $\overline{\boldsymbol{\omega}}_{2} \triangleq\left[\bar{\omega}_{n_{1}}, \bar{\omega}_{n_{2}}\right]^{T}$ with $\bar{\omega}_{n}$ to be complex. Once we find the optimum solution $\bar{\omega}_{2}^{*}$, based upon which the integer version $\omega_{2}^{*}$ will be determined. Moreover, without loss of generality, we drop the iteration index $(i)$ throughout the mathematical work in this section, and this is for the sake of notation simplicity.

Define a cost function

$$
\begin{aligned}
f\left(\overline{\boldsymbol{\omega}}_{2}\right) & \triangleq\left\|\overline{\mathbf{s}}-\alpha \widetilde{\mathbf{H}} \overline{\boldsymbol{\omega}}_{2}\right\|^{2} \\
& =\|\overline{\mathbf{s}}\|^{2}-2 \alpha \Re\left(\overline{\mathbf{s}}^{H} \widetilde{\mathbf{H}} \overline{\boldsymbol{\omega}}_{2}\right)+\alpha^{2}\left\|\widetilde{\mathbf{H}} \overline{\boldsymbol{\omega}}_{2}\right\|^{2},
\end{aligned}
$$

where $\Re(\cdot)$ denotes the real part of a complex value. Let $\partial f\left(\overline{\boldsymbol{\omega}}_{2}\right) / \partial \overline{\boldsymbol{\omega}}_{2}=0$; and we can immediately obtain a standard form of Wiener equation [27]

$$
\overline{\boldsymbol{\omega}}_{2}^{*}=\alpha^{-1}\left(\widetilde{\mathbf{H}}^{H} \widetilde{\mathbf{H}}\right)^{-1} \widetilde{\mathbf{H}}^{H} \overline{\mathbf{s}} .
$$

Hence, $\overline{\boldsymbol{\omega}}_{2}^{*}$ is the optimum of the continuous version, which minimizes the cost function (17). However, the optimum of the integer version (i.e., $\boldsymbol{\omega}_{2}^{*}$ ) is not as simple as

$$
\boldsymbol{\omega}_{2}^{*}=\operatorname{round}\left(\overline{\boldsymbol{\omega}}_{2}^{*}\right) \text {. }
$$

This is because the elements $\omega_{n_{1}}, \omega_{n_{2}}$ have to be jointly optimized, and the round operation in (20) forms decision in an individual manner. The optimum vector $\omega_{2}^{*}$ can be found by searching exhaustively within the range of $\left|\boldsymbol{\omega}_{2}-\operatorname{round}\left(\overline{\boldsymbol{\omega}}_{2}^{*}\right)\right| \leq$ d. However, this will cost the computational complexity in the square order (for the real version of $\boldsymbol{\omega}$ ) or four (for the complex version of $\boldsymbol{\omega})$. Next, we will show that the exhaustive search is actually not needed.

Theorem 1: Given $n \in\left\{n_{1}, n_{2}\right\}$ and

$$
\begin{gathered}
\delta_{n}^{\perp} \triangleq \Re\left(\operatorname{round}\left(\bar{\omega}_{n}\right)-\bar{\omega}_{n}\right), \\
\delta_{n} \triangleq \omega_{n}-\Re\left(\bar{\omega}_{n}\right),
\end{gathered}
$$

$\delta_{n_{2}}$ can be determined by

$$
\delta_{n_{2}}=\operatorname{round}\left(-\frac{\Re\left(\tilde{\mathbf{h}}_{n_{2}}^{H} \tilde{\mathbf{h}}_{n_{1}}\right) \delta_{n_{1}}}{\left\|\tilde{\mathbf{h}}_{n_{2}}\right\|^{2}}-\delta_{n_{2}}^{\perp}\right)+\delta_{n_{2}}^{\perp},
$$

and $\delta_{n_{1}}^{2}$ is upper bounded by

$$
\delta_{n_{1}}^{2} \leq\left(\delta_{n_{1}}^{\perp}\right)^{2}+\frac{\left\|\tilde{\mathbf{h}}_{n_{2}}\right\|^{4}}{4\left(\left\|\tilde{\mathbf{h}}_{n_{1}}\right\|^{2}\left\|\tilde{\mathbf{h}}_{n_{2}}\right\|^{2}-\Re\left(\tilde{\mathbf{h}}_{n_{2}}^{H} \tilde{\mathbf{h}}_{n_{1}}\right)^{2}\right)},
$$

where $\tilde{\mathbf{h}}_{n}, n \in\left\{n_{1}, n_{2}\right\}$, are the two column vectors of $\widetilde{\mathbf{H}}$.

Proof: See Appendix.

Theorem 1 shows that $\omega_{n_{2}}^{*}$ is uniquely determined given $\omega_{n_{1}}^{*}$. Moreover, the term $\left(\omega_{n_{1}}^{*}-\Re\left(\bar{\omega}_{n_{1}}\right)\right)^{2}=\delta_{n_{1}}^{2}$ is upper bounded by (24). Our computer simulations have shown that for most of cases $\delta_{n_{1}}^{2}$ only has one (around $79 \%$ ) or two candidates (around 20\%) within the range (24), and thus the computational complexity of finding the optimum $\omega_{n_{1}}^{*}$ is linear.

As a summary, the $i^{\text {th }}$ iteration of the optimum D2VP is described by

The $i^{\text {th }}$ Iterative Process of The Optimum D2VP

\section{Start}

Given the indices $n_{1}, n_{2}$, form the matrix $\widetilde{\mathbf{H}}$;

1. Let $\overline{\mathbf{s}}=\overline{\mathbf{s}}(i-1)$; and compute $\overline{\boldsymbol{\omega}}_{2}^{*}$ via (19);

2. Compute $\delta_{n_{1}}^{\perp}$ and $\delta_{n_{2}}^{\perp}$ via (21);

3. Compute the upper bound of $\delta_{n_{1}}^{2}$ via (24);

4. Given a candidate of $\delta_{n_{1}}$ within the range, compute the corresponding $\delta_{n_{2}}$;

5. Use $\delta_{n_{1}}, \delta_{n_{2}}$ to compute $\omega_{n_{1}}, \omega_{n_{2}}$ via (22);

6. Apply $\omega_{n_{1}}, \omega_{n_{2}}$ into (11) and find the best $\boldsymbol{\omega}_{2}^{*}(i)$.

Repeat the above until all states of $\left(n_{1}, n_{2}\right)$ are visited;

Find the optimum $\boldsymbol{\omega}_{2}^{*}(i)$ amongst all visited states of $\left(n_{1}, n_{2}\right)$. End

\section{D2VP with the complex version of $\omega$}

The optimum D2VP approach presented in Section III-B is for the case when the perturbation vector $\boldsymbol{\omega}$ is real. When $\boldsymbol{\omega}$ is complex, we can form an $N \times 2 N$ matrix $\mathbf{H}_{\mathrm{c}}=\left[\mathbf{H}^{\dagger}, j \mathbf{H}^{\dagger}\right]$ and a $2 N \times 1$ vector $\boldsymbol{\omega}_{\mathrm{c}}=\left[\Re(\boldsymbol{\omega})^{T}, \Im(\boldsymbol{\omega})^{T}\right]^{T}$, where $\Im(\cdot)$ denotes the imaginary part of a complex value. Then, the objective function (7) can be expressed by

$$
\boldsymbol{\omega}_{\mathrm{c}}^{*}=\underset{\boldsymbol{\omega}_{\mathrm{c}}}{\operatorname{argmin}}\left\|\overline{\mathbf{s}}-\alpha \mathbf{H}_{\mathrm{c}} \boldsymbol{\omega}_{\mathrm{c}}\right\|^{2} \text {. }
$$


Given $n_{1}, n_{2} \in[1,2 N]$, the matrix $\widetilde{\mathbf{H}}$ is formed by collecting the $n_{1}{ }^{\text {th }}$ and $n_{2}{ }^{\text {th }}$ column vector of $\mathbf{H}_{\mathrm{c}}$, and accordingly the perturbation vector $\boldsymbol{\omega}_{2}$ is formed by the $n_{1}{ }^{\text {th }}$ and $n_{2}{ }^{\text {th }}$ element of $\omega_{\mathrm{r}}$. Then, the objective function (11) still holds, and the optimum D2VP approach presented in Section III-B can be straightforwardly employed to obtain the optimum solution in the case of complex $\boldsymbol{\omega}$.

The only difference between the cases with complex and real version of $\boldsymbol{\omega}$ is: the complex version requires searching over $N(2 N-1)$ possible states of $\left(n_{1}, n_{2}\right)$, and this increases the computational complexity by around 3 folds. On the other hand, since $\omega_{\mathrm{c}}$ doubles the length of the perturbation vector, the error term $\varepsilon_{l}$ in (14)-(16) is largely reduced. Moreover, it is easy to understand that the real version is a subset of the complex version. Therefore, the complex version should outperform the real version in terms of the performance optimality, and this conclusion has been confirmed through the computer simulations (see Section V).

\section{Analysis of Computational Complexity}

The overall computational complexity of the optimum D2VP approach is easy to calculate. Take the real version of $\boldsymbol{\omega}$ as an example. For each iteration, the optimum D2VP approach spends the complexity of $\frac{N(N-1)}{2}$ for the exhaustive search over all possible states of $\left(n_{1}, n_{2}\right)$. Moreover, for each state, we need to search all candidates of $\delta_{n_{1}}$. Given the total number of $I$ iterations as well as the maximum of $\mathcal{L}$ candidates of $\delta_{n_{1}}$, the overall computational complexity is

$$
\frac{I \mathcal{L} N(N-1)}{2}
$$

However, (26) does not mean that the optimum D2VP approach requires the computational complexity in order of four. This is because the variables $I$ and $\mathcal{L}$ are not a function of $N$; and for most of the cases, we have $\mathcal{L} \leq 2$ and $I \leq 3$. Therefore, the computational complexity for the real version of optimum D2VP is only in the square order of the size of MU-MIMO, i.e., $\mathcal{O}(N(N-1))$. The same complexity order also holds for the complex version of D2VP. Specifically, it is given by $\mathcal{O}(N(2 N-1))$.

\section{COMPleXity-Reduced ApproACH OF D2VP}

Section III-D has shown that, apart from the inverse of the channel matrix, the major complexity of the optimum D2VP approach comes from searching over all possible states of $\left(n_{1}, n_{2}\right)$. However, due to the randomness of the channel matrix $\mathbf{H}$, it is unlikely to identify the best state of $\left(n_{1}, n_{2}\right)$ without employment of the exhaustive search. Therefore, the objective of this section is to propose a complex-reduced (CR) approach of D2VP, which can avoid the exhaustive search at the price of the performance.

Given the singular-value decomposition (SVD): $\mathbf{H}=$ $\mathbf{V}^{H} \boldsymbol{\Sigma} \mathbf{U}$, the SVD of $\mathbf{H}^{\dagger}$ is

$$
\mathbf{H}^{\dagger}=\mathbf{U}^{H} \boldsymbol{\Sigma}^{\dagger} \mathbf{V}
$$

where $\mathbf{U}, \mathbf{V}$ is unitary matrix, and $\boldsymbol{\Sigma}$ a diagonal matrix with the singular values of $\mathbf{H}$ on its diagonal. When $\mathbf{H}$ is generated

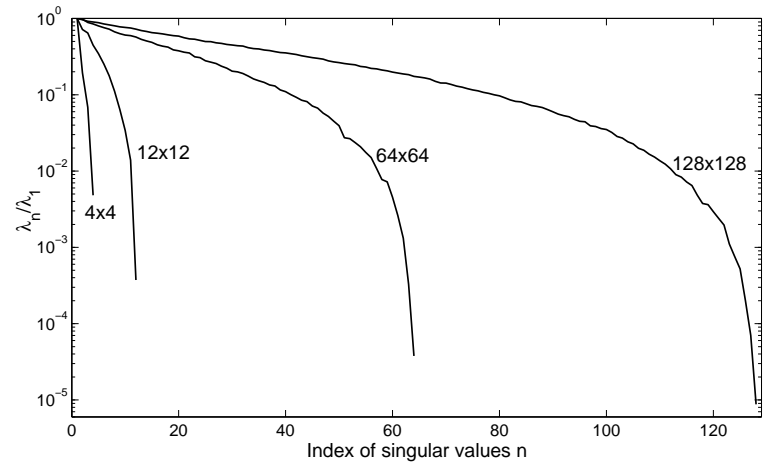

Fig. 1. Showcase the ratio of $\lambda_{n} / \lambda_{1}$ with respect to the size of MU-MIMO $(N=4,12,64,128)$.

according to the i.i.d. complex Gaussian distribution, $\boldsymbol{\Sigma}$ can have very small singular values, but the probability for a singular value to be zero is negligibly small. Hence, we have $\boldsymbol{\Sigma}^{\dagger}=\boldsymbol{\Sigma}^{-1}$ hold in general. With this fact in mind, we apply (27) into (7) and obtain

$$
\begin{aligned}
\boldsymbol{\omega}^{*} & \left.=\underset{\boldsymbol{\omega}}{\arg \min } \| \mathbf{U}^{H} \boldsymbol{\Sigma}^{-1} \mathbf{V}(\mathbf{s}-\alpha \boldsymbol{\omega})\right) \|^{2}, \\
& \left.=\underset{\boldsymbol{\omega}}{\operatorname{argmin}} \| \boldsymbol{\Sigma}^{-1} \mathbf{V}(\mathbf{s}-\alpha \boldsymbol{\omega})\right) \|^{2} .
\end{aligned}
$$

Denoting $\sqrt{1 / \lambda_{1}}, \ldots, \sqrt{1 / \lambda_{N}}$ to be the singular values of $\mathbf{H}$ $\left(\lambda_{1}>\lambda_{2}>\ldots>\lambda_{N}>0\right)$ and $\mathbf{v}_{n}^{T}$ the $n^{\text {th }}$ row vector of $\mathbf{V}$, (29) can be represented by

$$
\begin{aligned}
\boldsymbol{\omega}^{*} & =\underset{\boldsymbol{\omega}}{\operatorname{argmin}} \sum_{n=1}^{N} \lambda_{n}\left|\mathbf{v}_{n}^{T}(\mathbf{s}-\alpha \boldsymbol{\omega})\right|^{2}, \\
& =\underset{\boldsymbol{\omega}}{\operatorname{argmin}} \lambda_{1} \sum_{n=1}^{N}\left(\frac{\lambda_{n}}{\lambda_{1}}\right) \gamma_{n},
\end{aligned}
$$

where

$$
\gamma_{n} \triangleq\left|\mathbf{v}_{n}^{T} \mathbf{s}-\alpha \mathbf{v}_{n}^{T} \boldsymbol{\omega}\right|^{2}
$$

It is of our interest to study the ratio $\lambda_{n} / \lambda_{1}, \forall n$, with various configurations of the size of MU-MIMO, which is illustrated in Fig. 1. It is observed that the ratio $\lambda_{n} / \lambda_{1}$ drops rapidly with the increase of $n$. For most of the singular values, the ratio $\lambda_{n} / \lambda_{1}$ is negligibly small $(<0.1)$, and only a small portion of the singular values dominate the objective function (31). Therefore, we can form an approximate version of (31)

$$
\boldsymbol{\omega}^{*}=\underset{\boldsymbol{\omega}}{\operatorname{argmin}} \lambda_{1} \sum_{n=1}^{\bar{N}}\left(\frac{\lambda_{n}}{\lambda_{1}}\right) \gamma_{n},
$$

where $\bar{N}(<N)$ is the number of dominating singular values. Fig. 1 shows that $\bar{N}$ is quite small (around $1-3$ ) for the small and medium size of MU-MIMO (e.g. $N=4$ and $N=12$ ). On the other hand, it increases to a relatively large number for the large size of MU-MIMO (e.g. $\bar{N}>60$ for $N=128$ ).

In order to clarify the rationale of the CR-D2VP approach, our presentation starts with the small size of MU-MIMO (e.g. $N=4$ ), where we have $\bar{N}$ to be as small as 1 . In this case, 


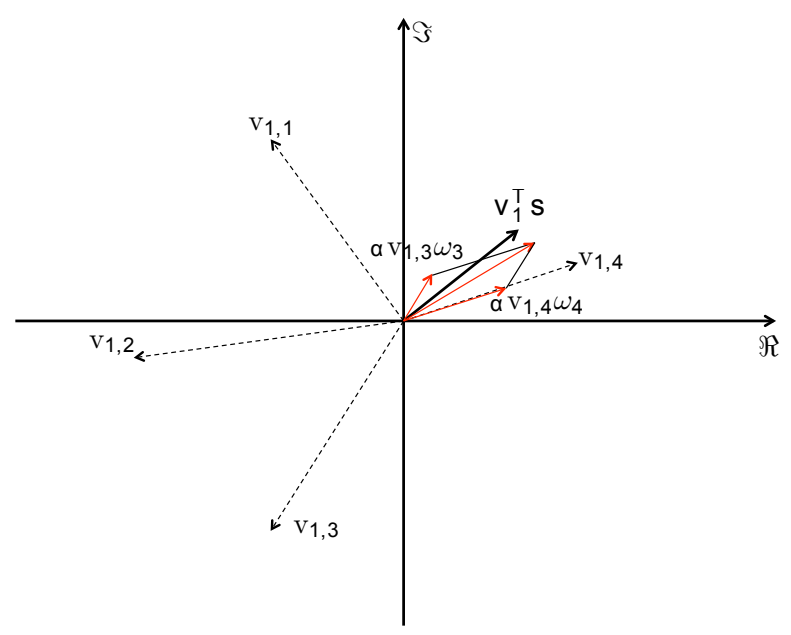

Fig. 2. An example of the proposed D2VP solution to (34).

if we can get $\gamma_{1}$ to be minimized, then the impact of $\lambda_{1}$ is minimized. Therefore, our objective here is

$$
\begin{aligned}
\boldsymbol{\omega}^{*} & =\underset{\boldsymbol{\omega}}{\operatorname{argmin}} \gamma_{1} \\
& =\underset{\boldsymbol{\omega}}{\operatorname{argmin}}\left|\mathbf{v}_{1}^{T} \mathbf{s}-\alpha \mathbf{v}_{1}^{T} \boldsymbol{\omega}\right|^{2} .
\end{aligned}
$$

Indeed, finding the optimum solution of (34) still costs the computational complexity in an exponential order. However, we are able to find a D2VP approach, which offers a suboptimum solution to (34).

Denote $\mathrm{v}_{1, n}$ to be the $n^{\text {th }}$ element of $\mathbf{v}_{1}$. Fig. 2 shows an example of $N=4$, where the elements of $\mathbf{v}_{1}$ are randomly distributed in a complex plane. Two of the elements (i.e., $\mathrm{v}_{1,3}$ and $\mathrm{v}_{1,4}$ ) are composed into a new complex, which is very close to the complex $\mathbf{v}_{1}^{T} \mathbf{s}$. In the procedure of complex composition, the two real integers $\omega_{3}$ and $\omega_{4}$ are utilized to scale the complex elements $\mathrm{v}_{1,3}$ and $\mathrm{v}_{1,4}$, respectively.

In terms of computing, there are many ways of selecting the two elements of $\mathbf{v}_{1}$ (or correspondingly the two elements of $\boldsymbol{\omega})$. A straightforward way is to exhaustively visit all possible combinations of any two components of $\mathbf{v}_{1}$. This of course leads to the best selection at the price of a square-order of the computational complexity, and thus the exhaustive search is not a favourable approach. Here, we propose a simple approach of selection.

Denote $\angle\left(\mathbf{v}_{1}^{T} \mathbf{s}\right)$ to be the phase of $\mathbf{v}_{1}^{T} \mathbf{s}$. The proposed approach first computes

$$
\psi_{n}= \begin{cases}\angle\left(\mathrm{v}_{1, n}\left(\mathbf{v}_{1}^{T} \mathbf{s}\right)^{H}\right), & n=1, \ldots, N \\ \angle\left(-\mathrm{v}_{1, n-N}\left(\mathbf{v}_{1}^{T} \mathbf{s}\right)^{H}\right), & n=N+1, \ldots, 2 N\end{cases}
$$

which includes the angles and complementary angles between $\mathrm{v}_{1, n}$ and $\mathbf{v}_{1}^{T} \mathbf{s}$. Then, we find the smallest positive value and the largest negative value of $\psi_{n}$. If these two values are corresponding to two different elements of $\mathbf{v}_{1, n}$, then we said that the desired elements have been found; or otherwise assuming $\psi_{1}(>0)$ and $\psi_{2}(<0)$ to be the angles of interest with $\left|\psi_{1}\right|>\left|\psi_{2}\right|$, we suggest to replace $\psi_{1}$ with the second smallest positive value of $\psi_{n}$. By this means, we are able to find two elements of $\mathbf{v}_{1}$ (or equivalently $\boldsymbol{\omega}$ ), which can form the complex composition as illustrated in Fig. 2. The proposed approach is clearly sub-optimum for a small $N$, and it tends to optimum for $N \rightarrow \infty$. This is because $\mathrm{v}_{1, n}$ is randomly distributed in the complex plane according to the standard Gaussian distribution. It is easy to find that the proposed approach costs only linear complexity.

As long as the two elements of $\boldsymbol{\omega}$ are selected, we can form the objective function (11) and determine $\boldsymbol{\omega}_{2}^{*}$ by employing Theorem 1. Finally, we have two remarks about the RC-D2VP approach:

Remark 1: Analogous to the optimum D2VP approach, the CR-D2VP approach can also be straightforwardly extended to the case of complex $\boldsymbol{\omega}$ through the objective function (25). Moreover, it is easy to justify that the complex version of CR-D2VP also features linear computational complexity.

Remark 2: So far, the discussion about CR-D2VP is for the small size of MU-MIMO. When the size of MU-MIMO becomes large (e.g. $N=64,128$ ), the objective function (34) would be over approximate. In this case, the CR-D2VP approach will suffer considerable optimality loss (this issue will be further examined through computer simulations). A potential way of improving the performance is to find the desired elements of $\boldsymbol{\omega}$ via

$$
\boldsymbol{\omega}^{*}=\underset{\boldsymbol{\omega}}{\arg \min }\left|\mathbf{v}_{n}^{T} \mathbf{s}-\alpha \mathbf{v}_{n}^{T} \boldsymbol{\omega}\right|^{2}, n=1, \ldots, \bar{N} .
$$

By this means, we will have at most $\bar{N}$ candidates of $\boldsymbol{\omega}_{2}$, with which Theorem 1 can be employed to determine the best one for performing the D2VP processing. However, the computational complexity of the RC-D2VP approach increases to $\mathcal{O}(\bar{N} N)$.

\section{Computer Simulations and Performance EVALUATION}

The primary objective of computer simulations is to examine the link-level scalability of the D2VP nonlinear precoding technique with respect to the size of MU-MIMO networks. The key performance metrics are the bit-error-rate (BER) performance and computational complexity. The baseline for performance comparison includes three techniques, which are the SE-VP, LR-VP, as well as the linear ZF precoding. The SEVP technique should offer the best performance for the small size of MU-MIMO (e.g. $N=4$ ). However, the computational complexity of SE-VP increases exponentially with respect to the size of MU-MIMO, and thus for the medium and large size of MU-MIMO (e.g. $N \geq 8$ ), we employ the LR-VP technique as the main baseline due to its well-recognized performance-complexity tradeoff. In terms of the performance, the linear ZF precoding surely performs the worst. However, it features the lowest computational complexity, and thus serves as an excellent baseline to evaluate the complexity cost of the D2VP technique. It is worthwhile to note that the V-BLAST based NLP technique is not employed for the performance comparison. This is mainly because the LR-VP technique has been proved to be better than the V-BLAST technique in terms of the performance [12], and we omit the V-BLAST results for the sake of delivering a concise presentation. 
In our computer simulations, the MU-MIMO system was configured exactly the same as that has been introduced in Section II. Each entry of the channel matrix $\mathbf{H}$ was independently generated according to the complex Gaussian distribution with normalised variance. We recognize the fact that the entries of $\mathbf{H}$ can be statistically correlated in practice, and the wireless communication channels are mostly frequency selective. However, we note that the channel correlation will only reduce the number of DoF in the spatial domain, and this is equivalent to the case of reducing the size of uncorrelated MIMO in our simulation model. Moreover, we assume that the frequency-selective channel can be nicely converted into a number of parallel flat sub-channels through employment of multi-carrier transmissions, and our interest is focused on one of the sub-channels. In fact, similar simulation setup has been widely adopted by most of previous works in this scope.

With the above system configuration, the BER results were obtained by taking an average over up to 400,000 independent channel realizations. The SNR is defined by the average received bit-energy per antenna to noise ratio. It is worthwhile to note that our simulations are mainly for an uncoded source with 4-QAM modulation (16-QAM will also be examined mainly for the sake of elaboration). This is because the VP technique has already been well evaluated for the coded sources and optimized for various order of QAM modulations (see [10]). Since the D2VP technique does not change the basic structure of VP, and our simulations are used mainly for the evaluation of large MU-MIMO systems, uncoded source is considered to be a more cost-effective option for the computer simulations.

Specifically, our computer simulations are structured into the following four experiments.

Experiment 1: The objective of this experiment is to examine the performance of the optimum D2VP approach when the perturbation vector $\boldsymbol{\omega}$ is complex. Fig. 3 illustrates the BER results as a function of $\mathrm{Eb} / \mathrm{No}$ for the case of $4 \times 4 \mathrm{MU}$ MIMO (i.e., $N=4$ ). Generally, the SE-VP approach gives the best BER performance; and there is a large SNR gap (10 $\mathrm{dB}$ difference in $\mathrm{Eb} / \mathrm{No}$ ) observed between the SE-VP and the linear ZF precoding. The LR-VP approach shows up to $2 \mathrm{~dB}$ difference in Eb/No in comparison with the SE-VP approach. The maximal gap appears at the medium SNRs $(\mathrm{Eb} / \mathrm{No}=8$ $10 \mathrm{~dB})$. Moreover, at the high SNR $(\mathrm{Eb} / \mathrm{No}=20 \mathrm{~dB})$, the LRVP approach performs even better than the SE-VP approach. This result is however not surprising. The major reason is that the SE-VP approach was searching for the optimum solution within a finite set of integers, i.e., $\{-j,-1,0,1, j\}$. Such approximation is acceptable at low and medium SNRs; however it results in considerable optimality loss at high SNRs.

Now, let us take a close look of the optimum D2VP approach. It is observed that the D2VP approach offers very close performance to the SE-VP at low and medium SNRs $(\mathrm{Eb} / \mathrm{No} \leq 10 \mathrm{~dB})$. Moreover, it outperforms the LR-VP approach by around $2 \mathrm{~dB}$ in Eb/No. However, when Eb/No is as high as $16 \mathrm{~dB}$ or above, D2VP performs worse than the LR-VP approach. This is because the D2VP approach only utilizes two subspaces per iteration for conducting the vector perturbation,

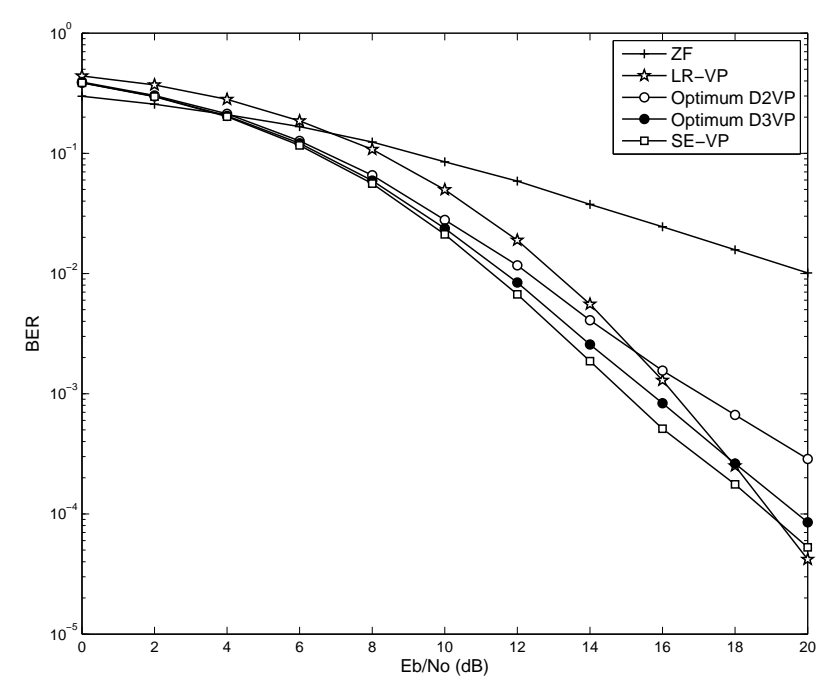

Fig. 3. BER performance of the optimum D2VP (complex version) with $N=4$ and 4-QAM.

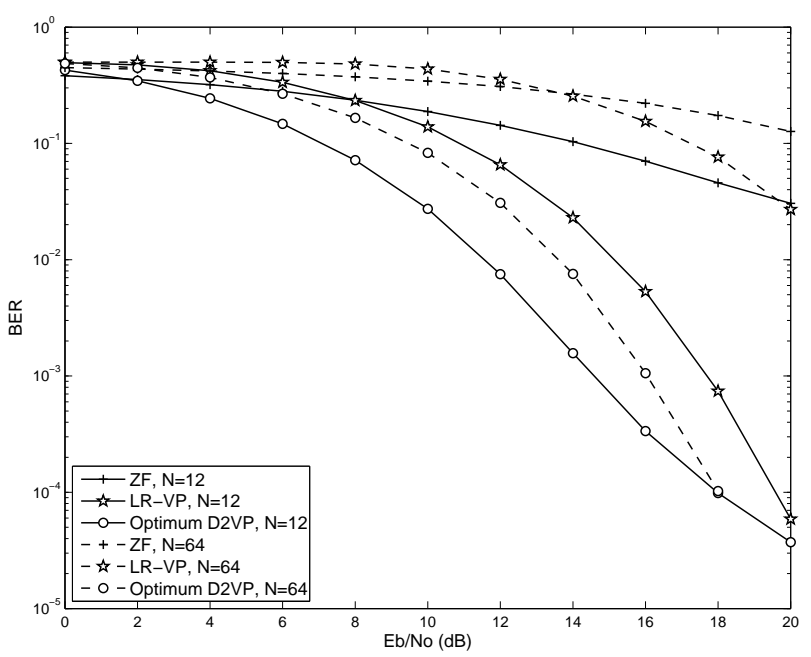

Fig. 4. BER performance of the optimum D2VP (complex version) with $N=12,64$ and 4-QAM.

which is too approximate at high SNRs. Nevertheless, we will show in Experiment 3 that the D2VP approach offers significant advantage in terms of the computational complexity.

It is worthwhile to highlight that the optimum D2VP approach offers comparable computational complexity to the linear ZF precoding. With this fact in mind, we can see that the optimum D2VP approach generally outperforms the ZF precoding by around $8 \mathrm{~dB}$ in Eb/No. In addition, we also examined the performance of using degree- 3 vector perturbation (D3VP), which shares the same principle as the D2VP at the cost of much higher computational complexity. It is observed that the D3VP approach further improves the BER performance by around $2 \mathrm{~dB}$ at high SNRs (Eb/No $\geq 18 \mathrm{~dB})$. This performance gain mainly comes from less approximation used in the D3VP approach. 


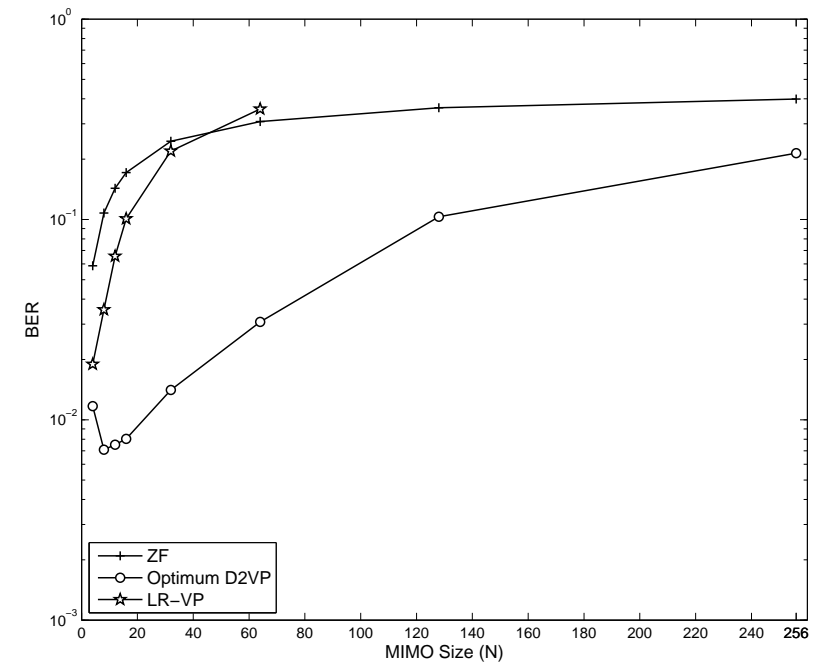

Fig. 5. BER performance of 4-QAM modulated MIMO with various MIMO size at $\mathrm{Eb} / \mathrm{No}=12 \mathrm{~dB}$.

Fig. 4 illustrates the BER results of various techniques for the examples of medium and large size of MU-MIMO ( $N=12$ and 64$)$. The performance of SE-VP approach is not provided due to its extremely high computational complexity. Generally, all of the demonstrated approaches get their BER performances worse when the size of MU-MIMO becomes large. The optimum D2VP approach gets less performance reduction in comparison with the others, and it shows the best performance at all SNRs. Specifically, the optimum D2VP approach outperforms the LR-VP approach by up to $4 \mathrm{~dB}$ for the case of $N=12$ and $8 \mathrm{~dB}$ for the case of $N=64$, respectively. It is also observed that the LR-VP approach does not perform well for the lager size of MU-MIMO $(N=64)$, where its performance is even worse than the ZF precoding at low SNRs. This result is reasonable since the modulo receiver (4) is very sensitive to the poor vector perturbation particularly at low SNRs.

By fixing $\mathrm{Eb} / \mathrm{No}=12 \mathrm{~dB}$ (the typical configuration for mobile systems), Fig. 5 demonstrates the BER performance as a function of the size of MU-MIMO (i.e., $N$ ). It is observed that, for $N \leq 64$, both the LR-VP and ZF precoding get their performances rapidly degraded with the increase of $N$. For the case of $N>64$, we can still observe slight performance degradation of the ZF precoding. The performance of LR-VP is not provided due to its very high computational complexity in the case of large MU-MIMO. Interestingly, we find that the performance of D2VP gets improved when $N$ increases from 4 to 8 . It indicates that the optimum D2VP approach is able to enjoy the spatial-domain diversity gain in the case of small MU-MIMO. On the other hand, D2VP also shows quick performance degradation for the medium and large size of MU-MIMO. Nevertheless, D2VP always shows large performance improvement in comparison to the other techniques.

The last result of Experiment 1 shows the statistics of the vector perturbation, which is provided in TABLE I. It is found
TABLE I

STATISTICS OF VECTOR PERTURBATION OF THE OPTIMUM D2VP AND SE-VP WITH 4-QAM MODULATION.

\begin{tabular}{|c|c|c|c|c|c|}
\hline \multirow{2}{*}{ Probability } & \multicolumn{5}{|c|}{$\omega_{n}$} \\
\cline { 2 - 6 } & -2 & -1 & 0 & 1 & 2 \\
\hline D2VP, $N=4$ & $0.1 \%$ & $7.8 \%$ & $84 \%$ & $7.8 \%$ & $0.1 \%$ \\
\hline D2VP, $N=64$ & $0.3 \%$ & $2.6 \%$ & $94 \%$ & $2.6 \%$ & $0.3 \%$ \\
\hline SE-VP, $N=4$ & $0.1 \%$ & $10.2 \%$ & $79 \%$ & $10.2 \%$ & $0.1 \%$ \\
\hline
\end{tabular}

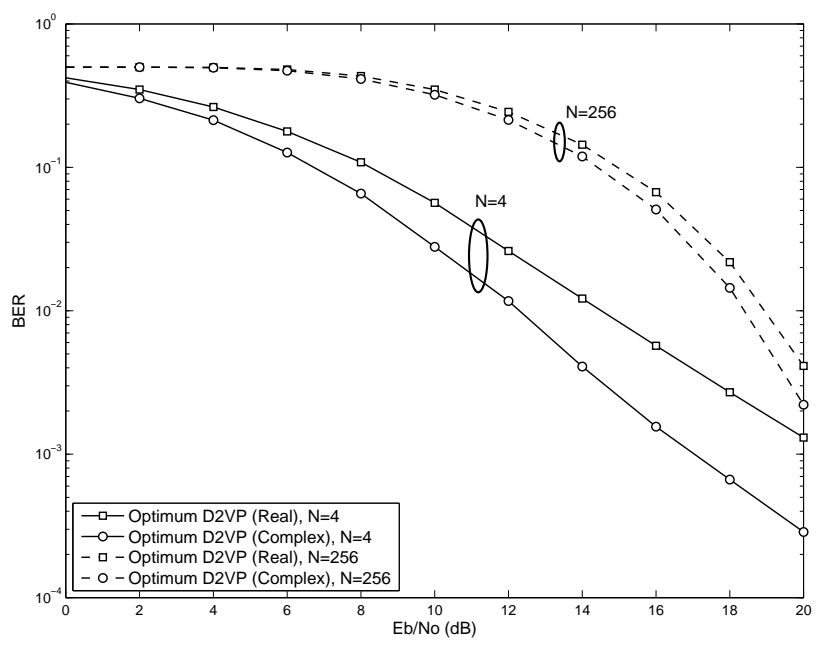

Fig. 6. Comparison of BER performances between the optimum D2VP approaches with complex and real perturbation.

that the SE-VP approach has around $79 \%$ of cases, which do not need vector perturbation. This statistical result confirms the sparse nature of the perturbation vector. Moreover, it also means that, for the small size of MU-MIMO $(N=4)$, we have around $79 \%$ of the cases where the ZF precoding is close to the optimum. This is actually a well-recognized conclusion in the literature (see [22]). For the optimum D2VP approach with $N=4$, we can find by around $84 \%$ of the cases where the vector perturbation is not needed. This result is quite close to that of SE-VP. When the size of MU-MIMO becomes large (e.g. $N=64$ ), the percentage of cases without vector perturbation increases to $94 \%$. This statistical result indicates the optimality loss of D2VP in the large size of MU-MIMO.

Experiment 2: The objective of this experiment is to examine the performance of optimum D2VP when the perturbation vector $\boldsymbol{\omega}$ is real. Fig. 6 demonstrates the performance difference between the real version and complex version of the optimum D2VP approach for both the small $(N=4)$ and large ( $N=256$ ) size of MU-MIMO. It is observed that the real version is about $3.5 \mathrm{~dB}$ worse than the complex version for the small size of MU-MIMO, and the difference becomes about $0.5 \mathrm{~dB}$ for the large size of MU-MIMO. This phenomenon is exactly in line with our theoretical analysis in Section III.

Fig. 7 exhibits the performance difference between the real and complex version of the optimum D2VP approach for various configurations of $N$ with $\mathrm{Eb} / \mathrm{No}=12 \mathrm{~dB}$. Generally, the performance difference is relatively large for the small size of MU-MIMO, and it mitigates with the increase of $N$. Moreover, it is observed that the real version also enjoys the 


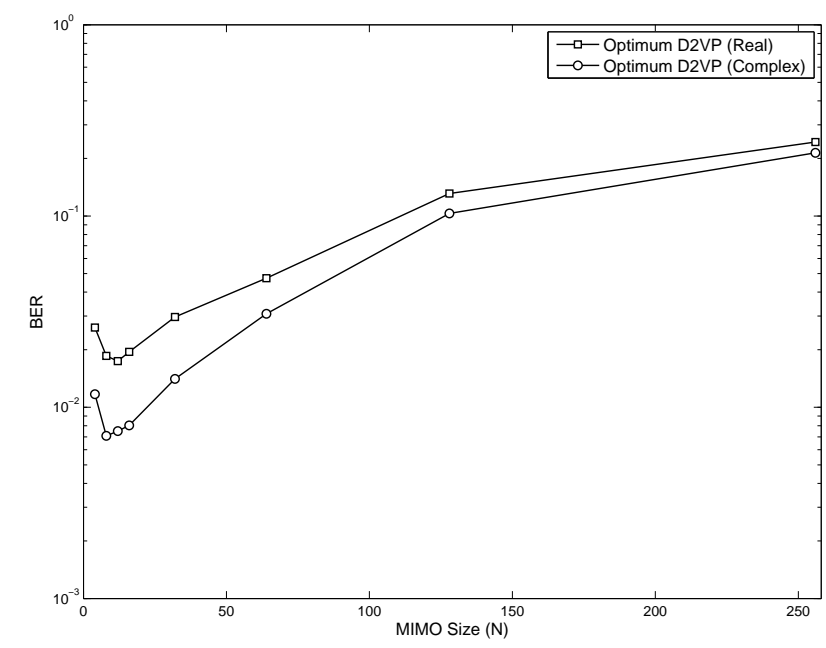

Fig. 7. Comparison of BER performances between the optimum D2VP approaches with complex and real perturbation at $\mathrm{Eb} / \mathrm{No}=12 \mathrm{~dB}$.

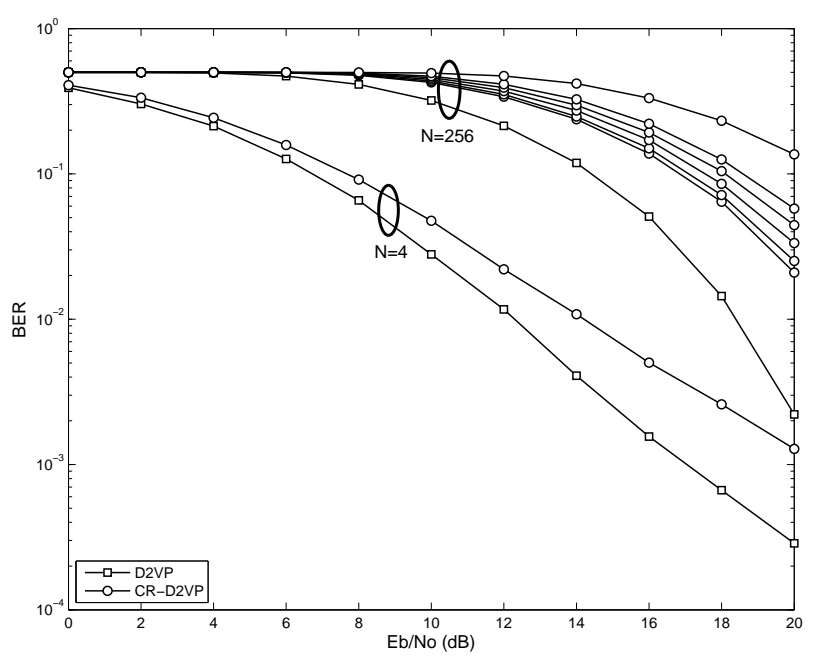

Fig. 8. Comparison of BER performances between the optimum D2VP

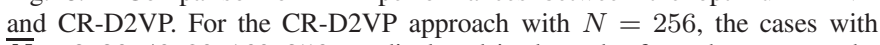
$\bar{N}=2,20,40,80,160,256$ are displayed in the order from the worst to the best.

spatial-domain diversity gain in the case of small MU-MIMO.

Experiment 3: The objective of this experiment is to examine the performance of CR-D2VP as well as the performancecomplexity tradeoff of various VP approaches. The performance comparison between the CR-D2VP and optimum D2VP is provided in Fig. 8. Both approaches use the complex vector perturbation. For the case of $N=4$, the CR-D2VP approach (with $\bar{N}=2$ ) shows relatively close performance to the optimum D2VP approach particularly for the low and medium $\mathrm{SNR}$ range $(\mathrm{Eb} / \mathrm{No}<12 \mathrm{~dB})$. For the case of $N=256$, the CR-D2VP approach (with $\bar{N}=2$ ) shows about $6 \mathrm{~dB}$ SNR gap when comparing to the optimum D2VP approach. It is clear that the configuration of $\bar{N}=2$ is too approximate in terms of the performance optimality. Surely,

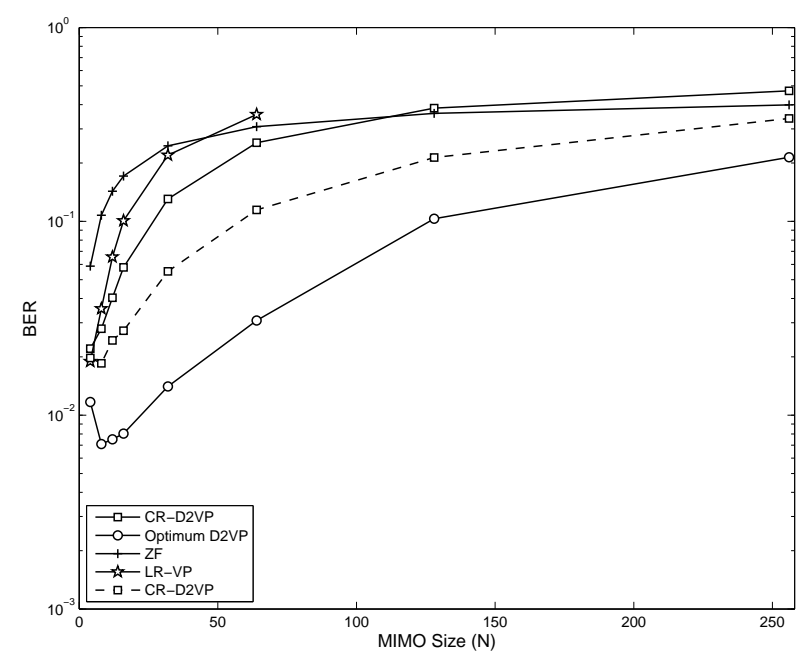

Fig. 9. Comparison of BER performances between the optimum D2VP and CR-D2VP at $\mathrm{Eb} / \mathrm{No}=12 \mathrm{~dB}$. RC-D2VP has two results: the solid line is for $\bar{N}=2$, and the dash line is for $\bar{N}=N$.

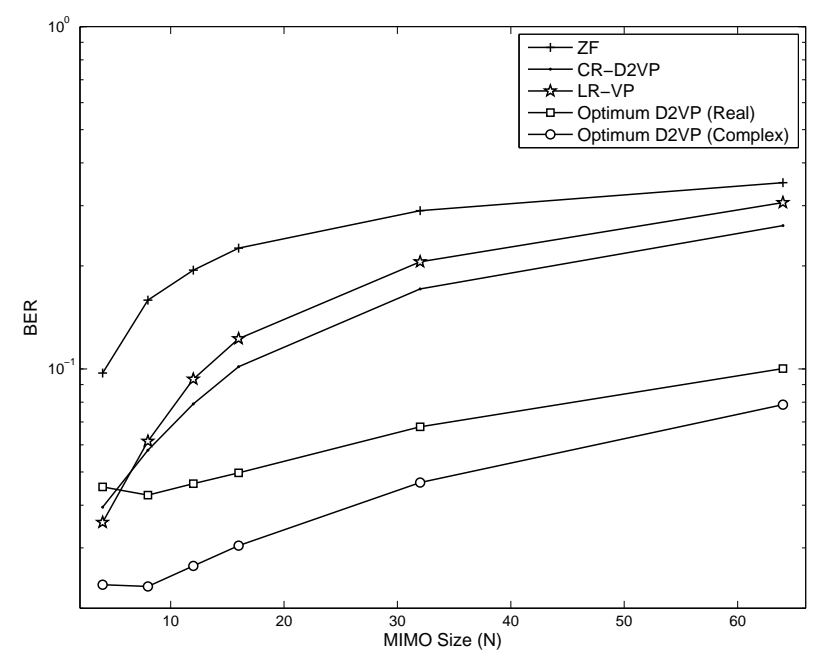

Fig. 10. BER performance of 16-QAM modulated MU-MIMO with various $N$ at $\mathrm{Eb} / \mathrm{No}=12 \mathrm{~dB}$.

the performance of CR-D2VP can be improved by increasing $\bar{N}$ at the price of linearly increased computational complexity. However, the largest performance improvement (about $2 \mathrm{~dB}$ in Eb/No) appears when $\bar{N}$ increases from 2 to 20 . Further 2 $\mathrm{dB}$ performance improvement is observed when $\bar{N}$ increases from 20 to 256 .

Fig. 9 shows the performance of CR-D2VP as a function of $N$ at $\mathrm{Eb} / \mathrm{No}=12 \mathrm{~dB}$. Generally, CR-D2VP outperforms the LR-VP approach. Unlike the optimum D2VP, it is found that the CR-D2VP approach does not enjoy spatial-domain diversity gain. In addition, the CR-D2VP approach with $\bar{N}=$ 2 performs slightly worse than the ZF precoding at high SNRs. Therefore, we suggest to employ a larger $\bar{N}$ when adopting the CR-D2VP approach at high SNRs.

So far, all of the simulation results are for 4-QAM mod- 


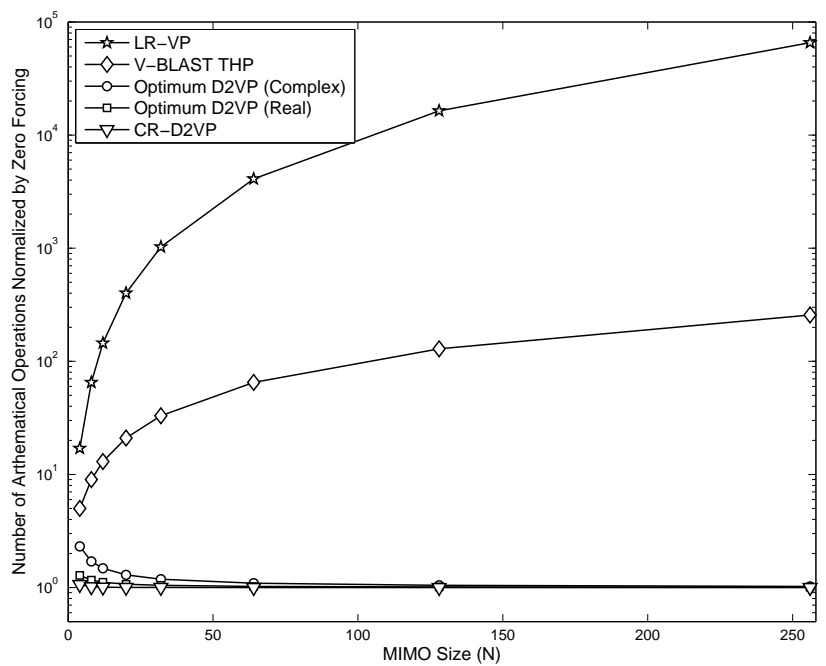

Fig. 11. Comparison of the computational complexity for various precoding approaches with respect to the size of MU-MIMO.

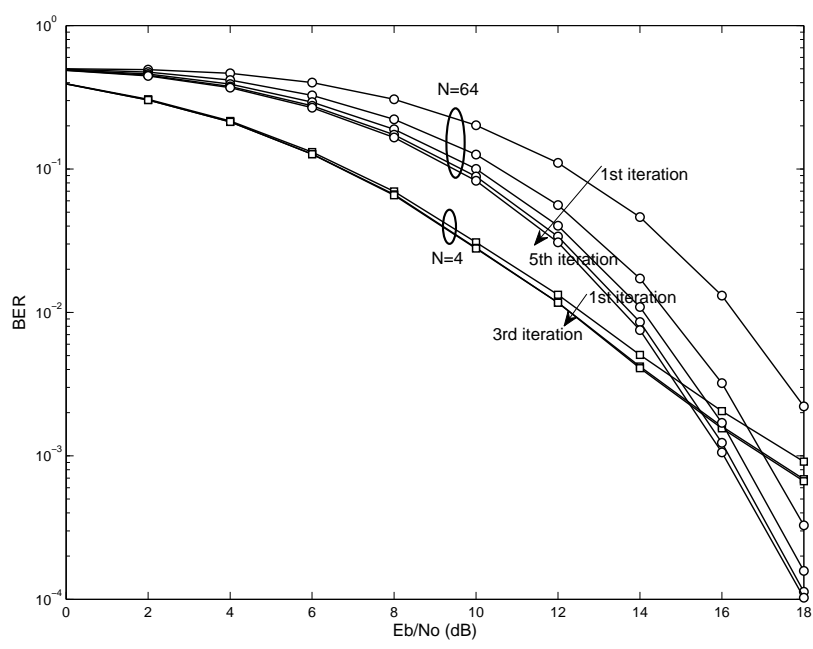

Fig. 12. BER performance of the optimum D2VP with various number of iterations.

ulation. It is probably also interesting to see how the D2VP approaches behaviour for higher-order modulations. Since the optimum configuration of VP with respect to the QAM modulation schemes has been well studied in [10], here we pick up 16-QAM as a showcase to demonstrate the BER performance as a function of $N(\mathrm{~Eb} / \mathrm{No}=12 \mathrm{~dB})$. The simulation results are depicted in Fig. 10. It is observed that the D2VP approaches significantly outperform the LR-VP approach. Particularly, the optimum D2VP can largely improve the BER performance for the case of $N \geq 12$. Fig. 11 shows the computational complexity of NLP techniques, which is normalized by the complexity of ZF precoding. It is shown that the D2VP approaches can significantly reduce the computational complexity of NLP. The complexity reduction is about $10-50 \mathrm{~dB}$ (subject to the size of MU-MIMO) when comparing with the LR-VP approach.

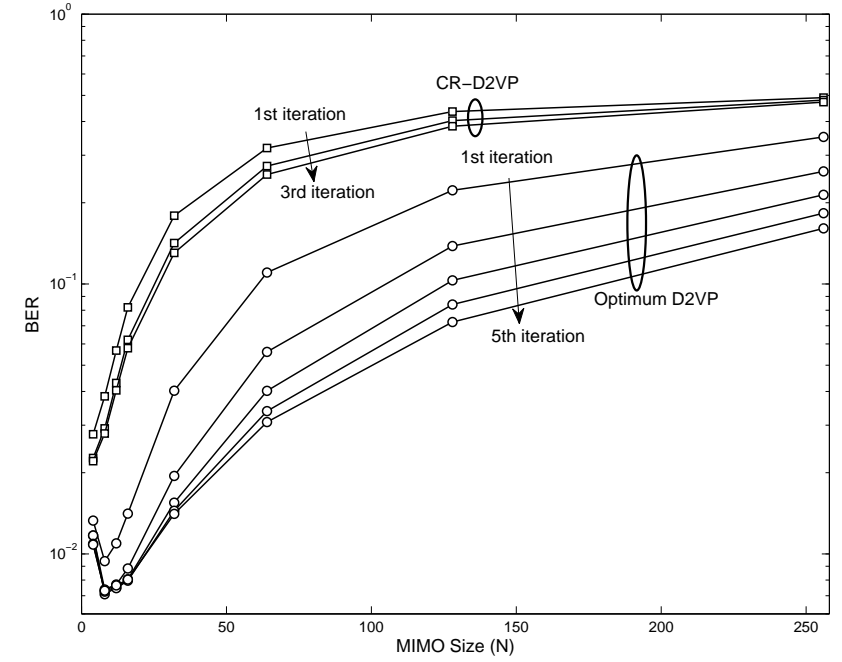

Fig. 13. Convergence study of the optimum D2VP and CR-D2VP for various size of MU-MIMO at $\mathrm{Eb} / \mathrm{No}=12 \mathrm{~dB}$.

More interestingly, the D2VP approaches show almost similar complexity as the ZF precoding when the size of MU-MIMO becomes large (e.g. $N \geq 12$ ). This is because, in the case of large MU-MIMO, the computational complexity of D2VP is dominated by the operation of channel matrix inverse.

Experiment 4: The objective of this experiment is to examine the convergence behaviour of the D2VP approaches. Fig. 12 shows the BER performance of the optimum D2VP (complex version) with respect to the number of iterations. For the case of $N=4$, the performance does not get improved after just two iterations. The difference between the 1st iteration and the 2nd iteration is negligibly small. For the case of $N=64$, the performance of D2VP does not get considerably improved after three iterations.

Fig. 13 demonstrates the convergence behaviour of both the CR-D2VP $(\bar{N}=2)$ and optimum D2VP for various cases of $N$. The CR-D2VP approach shows the performance converged after one or two iterations. The optimum D2VP gets its performance considerably improved by employing three iterations. For the case of large MU-MIMO (e.g. $N \geq 128$ ), more iterations can further improve the performance of the optimum D2VP approach, although the improvement is not as large as the first three iterations. When the size of MUMIMO is relatively small (e.g. $N \leq 64$ ), the performance improvement is not considerable after three iterations.

\section{CONCLUSION}

In this paper, we have presented a novel multi-antenna nonlinear precoding technique, which demonstrated excellent performance and complexity scalability to the size of MUMIMO networks. By exploiting the sparse nature of the perturbation vector, the proposed technique tackles the integer leastsquare optimization problem through several iterations, with each performs degree-2 vector perturbation. By this means, the $N$-dimensional ILS optimization problem is effectively 
converted into a serial of 2-dimensional ILS optimization problems, each having a closed-form solution. Such an approach is called the optimum D2VP, which outperforms the LR-VP approach by $5-10 \mathrm{~dB}$ in terms of $\mathrm{Eb} / \mathrm{No}$ whilst providing $10-50 \mathrm{~dB}$ reduction in terms of the computational complexity. When comparing with the linear ZF precoding technique, the optimum D2VP demonstrates very competitive complexity cost particularly for the medium and large size of MU-MIMO (e.g. $N \geq 16$ ), and at the meantime it easily outperforms the $\mathrm{ZF}$ technique by $10 \mathrm{~dB}$ or more in Eb/No. In addition, we have also proposed a complexity-reduced version of D2VP in order to further reduce the computational complexity. The performance-complexity tradeoff of the complexity reduced version has been thoroughly justified and also evaluated through computer simulations.

We believe that the proposed D2VP approach can largely mitigate the problem of signal-processing scalability, and it could bring the multi-antenna nonlinear precoding technique much closer to the super-fast downlink application use-cases of $5 \mathrm{G}$ mobile wireless internet.

\section{APPENDIX A}

ProOF OF THEOREM 1

Define a cost function

$$
g\left(\boldsymbol{\omega}_{2}\right) \triangleq\left(f\left(\boldsymbol{\omega}_{2}\right)-f\left(\overline{\boldsymbol{\omega}}_{2}^{*}\right)\right) / \alpha^{2},
$$

here we use a complex form of $\boldsymbol{\omega}_{2}$ for notation simplicity. It is easy to understand

- $g\left(\boldsymbol{\omega}_{2}\right)>0$

- If there exists an optimum vector $\boldsymbol{\omega}_{2}^{*}$ which minimizes $f\left(\boldsymbol{\omega}_{2}\right)$, it will also minimize $g\left(\boldsymbol{\omega}_{2}\right)$.

Then, we apply (18) into (37) and obtain

$$
\begin{aligned}
g\left(\boldsymbol{\omega}_{2}\right)=- & 2 \alpha^{-1} \Re\left(\overline{\mathbf{s}}^{H} \widetilde{\mathbf{H}}\left(\boldsymbol{\omega}_{2}-\overline{\boldsymbol{\omega}}_{2}^{*}\right)\right) \\
& +\boldsymbol{\omega}_{2}^{H} \widetilde{\mathbf{H}}{ }^{H} \widetilde{\mathbf{H}} \boldsymbol{\omega}_{2}-\left(\overline{\boldsymbol{\omega}}_{2}^{*}\right)^{H} \widetilde{\mathbf{H}}^{H} \widetilde{\mathbf{H}} \overline{\boldsymbol{\omega}}_{2}^{*} .
\end{aligned}
$$

Further, we define $\delta \triangleq \omega_{2}-\overline{\boldsymbol{\omega}}_{2}^{*}$ and apply (19) into (38). It is tedious but straightforward to have

$$
g(\boldsymbol{\delta})=\boldsymbol{\delta}^{H} \widetilde{\mathbf{H}}^{H} \widetilde{\mathbf{H}} \boldsymbol{\delta} .
$$

The above derivation holds generally for the complex form. When $\delta$ takes the real form $\delta \triangleq\left[\delta_{n_{1}}, \delta_{n_{2}}\right]^{T}$, (39) can be expressed by

$$
g(\boldsymbol{\delta})=\left\|\tilde{\mathbf{h}}_{n_{1}}\right\|^{2} \delta_{n_{1}}^{2}+\left\|\tilde{\mathbf{h}}_{n_{2}}\right\|^{2} \delta_{n_{2}}^{2}+2 \Re\left(\tilde{\mathbf{h}}_{n_{2}}^{H} \tilde{\mathbf{h}}_{n_{1}}\right) \delta_{n_{1}} \delta_{n_{2}}
$$

Given $\delta_{n_{1}}, g(\boldsymbol{\delta})$ is a quadratic form of $\delta_{n_{2}}$. If $\delta_{n_{2}}$ could take an arbitrary value, then (40) achieves its minimum at

$$
\delta_{n_{2}}=-\frac{\Re\left(\tilde{\mathbf{h}}_{n_{2}}^{H} \tilde{\mathbf{h}}_{n_{1}}\right) \delta_{n_{1}}}{\left\|\tilde{\mathbf{h}}_{n_{2}}\right\|^{2}} .
$$

However, it is not the case. Therefore, $\delta_{n_{2}}$ takes the value specified in (23), with which we can ensure that $\left(\delta_{n_{2}}+\Re\left(\bar{\omega}_{n_{2}}\right)\right)$ is the best integer.

Then, we plug (23) into (40) in order to obtain $g(\boldsymbol{\delta})$ as a function only of $\delta_{n_{1}}$. However, (23) is not mathematically tractable. Hence, we redefine $\delta_{n_{2}}$ into

$$
\delta_{n_{2}}=-\frac{\Re\left(\tilde{\mathbf{h}}_{n_{2}}^{H} \tilde{\mathbf{h}}_{n_{1}}\right) \delta_{n_{1}}}{\left\|\tilde{\mathbf{h}}_{n_{2}}\right\|^{2}}+\theta_{n_{2}}\left(\delta_{n_{1}}\right)
$$

where $\theta_{n_{2}}\left(\delta_{n_{1}}\right) \in[-0.5,0.5]$ is the difference between (23) and (41). Plugging (42) into (40) results in

$g\left(\delta_{n_{1}}\right)=\frac{\left(\left\|\tilde{\mathbf{h}}_{n_{1}}\right\|^{2}\left\|\tilde{\mathbf{h}}_{n_{2}}\right\|^{2}-\Re\left(\tilde{\mathbf{h}}_{n_{2}}^{H} \tilde{\mathbf{h}}_{n_{1}}\right)^{2}\right) \delta_{n_{1}}^{2}}{\left\|\tilde{\mathbf{h}}_{n_{2}}\right\|^{2}}+\left\|\tilde{\mathbf{h}}_{n_{2}}\right\|^{2} \theta_{n_{2}}^{2}\left(\delta_{n_{1}}\right)$.

Now, our objective is to minimize (43) by configuring of $\delta_{n_{1}}$. It is observed that the first term at the right hand of (43) monotonically increases with respect to $\delta_{n_{1}}^{2}$, and the second term falls into the range of $\left[0, \frac{\left\|\tilde{\mathbf{h}}_{n_{2}}\right\|^{2}}{4}\right]$.

Denote $\left(\delta_{n_{1}}^{\top}\right)^{2}$ to be the upper bound of $\delta_{n_{1}}^{2}$. If (43) reaches its minimum at $\delta_{n_{1}}^{2}=\left(\delta_{n_{1}}^{\top}\right)^{2}$, then the minimum of $g\left(\delta_{n_{1}}\right)$ is lower bounded by

$$
g\left(\delta_{n_{1}}\right) \geq \frac{\left(\left\|\tilde{\mathbf{h}}_{n_{1}}\right\|^{2}\left\|\tilde{\mathbf{h}}_{n_{2}}\right\|^{2}-\Re\left(\tilde{\mathbf{h}}_{n_{2}}^{H} \tilde{\mathbf{h}}_{n_{1}}\right)^{2}\right)\left(\delta_{n_{1}}^{\top}\right)^{2}}{\left\|\tilde{\mathbf{h}}_{n_{2}}\right\|^{2}} .
$$

Moreover, (21) gives the minimum of $\delta_{n_{1}}^{2}$, which is $\left(\delta_{n_{1}}^{\perp}\right)^{2}$. Applying $\delta_{n_{1}}^{2}=\left(\delta_{n_{1}}^{\perp}\right)^{2}$ into (43), we obtain the upper bounded of $g\left(\delta_{n_{1}}\right)$

$g\left(\delta_{n_{1}}\right) \leq \frac{\left(\left\|\tilde{\mathbf{h}}_{n_{1}}\right\|^{2}\left\|\tilde{\mathbf{h}}_{n_{2}}\right\|^{2}-\Re\left(\tilde{\mathbf{h}}_{n_{2}}^{H} \tilde{\mathbf{h}}_{n_{1}}\right)^{2}\right)\left(\delta_{n_{1}}^{\perp}\right)^{2}}{\left\|\tilde{\mathbf{h}}_{n_{2}}\right\|^{2}}+\frac{\left\|\tilde{\mathbf{h}}_{n_{2}}\right\|^{2}}{4}$.

Hence, the lower bound in (44) has to be no larger than the upper bound in (45). By solving this inequality we will get the upper bound in (24), and Theorem 1 is therefore proved.

\section{REFERENCES}

[1] R. Tafazolli and L. Herault, "Europe 5G public private partnership programme: strategic research and innovation agenda "internet on the move"', Expert Advisory Group, 2013.

[2] I. E. Telatar, "Capacity of multi-antenna Gaussian channels", European Trans. Telecommun., vol. 10, no. 6, pp. 585-595, 1999.

[3] G. Caire and S. Shamai, "On the achievable throughput of a multiantenna Gaussian broadcast channel", IEEE Trans. Inf. Theory, vol. 49 no. 7, pp. 1691-1706, Jul. 2003.

[4] S. Vishwannath, N. Jindal, and A. Goldsmith, "Duality, achievable rates and sum-rate capacity of Gaussian MIMO broadcast channels", IEEE Trans. Inf. Theory, vol. 49, no. 10, pp. 2658-2668, Oct. 2003.

[5] R. Zamir, S. Shamai, and U. Erez, "Nested linear/lattice codes for structured multiterminal binning", IEEE Trans. Inf. Theory, vol. 48, no. 6, pp. 1250-1276, Jun. 2002.

[6] U. Erez, S. Shamai, and R. Zamir, "Capacity and lattice strategies for canceling known interference", IEEE Trans. Inf. Theory, vol. 51, no. 11 , pp. 3820-3833, Nov. 2005.

[7] W. Yu, D. P. Varodayan, and J. M. Cioffi, "Trellis and convolutional precoding for transmitter-based interference presubtraction", IEEE Trans. Commun., vol. 53, no. 7, pp. 1220-1230, Jul. 2005.

[8] G. J. Foschini, G. D. Golden, R. A. Valenzuela, and P. W. Wolniansky, "Simplified processing for high spectral efficiency wireless communication employing multi-element arrays", IEEE J. Sel. Areas Commun., vol. 17, no. 11, pp. 1841-1852, Nov. 1999.

[9] C. B. Peel, B. M. Hochwald, and A. L. Swindlehurst, "A vectorperturbation technique for near-capacity multiantenna multiuser communication - Part I: Channel inversion and regularization", IEEE Trans. Commun., vol. 53, no. 1, pp. 195-202, Jan. 2005.

[10] C. B. Peel, B. M. Hochwald, and A. L. Swindlehurst, "A vectorperturbation technique for near-capacity multiantenna multiuser communication - Part II: Perturbation", IEEE Trans. Commun., vol. 53, no. 3, pp. 537-544, Mar. 2005.

[11] E. Agrell, T. Eriksson, A. Vardy, and K. Zeger, "Closest point search in lattices", IEEE Trans. Inf. Theory, vol. 48, no. 8, pp. 2201-2214, Aug. 2002.

[12] C. Windpassinger, R. F. H. Fischer, and J. B. Huber, "Lattice-reductionaided broadcast precoding", IEEE Trans. Commun., vol. 52, no. 12, pp. 2057-2060, Dec. 2004. 
[13] D. Castanheira, A. Silva, and A. Gameiro, "Linear and nonlinear precoding schemes for centralized multicell MIMO-OFDM systems", Int. J. Wireless Personal Commun., vol. 72, no. 1, pp. 759-777, Sep. 2013.

[14] H-S. Han, S-H. Park, S. Lee, and I. Lee, "Modulo loss reduction for vector perturbation systems", IEEE Trans. Commun., vol. 58, no. 1, pp. 3392-3396, Dec. 2010.

[15] T. Yoo and A. Goldsmith, "On the optimality of multiantenna broadcast scheduling using zero-forcing beamforming", IEEE J. Sel. Areas Commun., vol. 24, no. 3, pp. 528-541, Mar. 2006.

[16] J. Hou, Y. Ma, N. Yi, and R. Tafazolli, "Reduced-complexity coordinated beamforming for multicell downlink max-min SINR problem", IEEE Wireless Commun. Letts., vol. 48, no. 8, pp. 353-356, Aug. 2014.

[17] M. O. Damen, H. El Gamal, and G. Caire, "On maximum-likelihood detection and the search for the closet lattice point", IEEE Trans. Inf Theory, vol. 49, no. 10, pp. 2389-2402, Oct. 2003.

[18] F. Kaltenberger, H. Jiang, and M. G. G. Knopp, "Relative channel reciprocity calibration in MIMO/TDD systems", in Proc. Future Network and Mobile Summit, Jun. 2010, vol. 1, pp. 1-10.

[19] T. Yoo, N. Jindal, and A. Goldsmith, "Multi-antenna downlink channels with limited feedback and user selection", IEEE J. Sel. Areas Commun., vol. 25, no. 7, pp. 1478-1491, 2007.

[20] R. Tandon, S. Mohajer, H. V. Poor, and S. Shamai, "Feedback and delayed CSI can be as good as perfect CSI", in Proc. ICC, Jun. 2012, vol. 1, pp. 2355-2359.

[21] J. Hou, N. Yi, and Y. Ma, "Joint space-frequency user scheduling for MIMO random beamforming with limited feedback", IEEE Trans. Commun., vol. 63, no. 6, pp. 2224-2236, Jun. 2015.

[22] D. Gesbert, S. Hanly, H. Huang, S. Shamai, O. Simeone, and W. Yu, "Multi-cell MIMO cooperative networks: A new look at interference", IEEE J. Sel. Areas Communs., vol. 28, no. 9, pp. 1380-1408, Dec. 2010

[23] S. Nanda, K. Balachandran, and S. Kumar, "Adaptation techniques in wireless packet data services", IEEE Commun. Mag., vol. 38, no. 1, pp. $54-64,2000$.

[24] A. Ghosh, R. Ratasuk, B. Mondal, N. Mangalvedhe, and T. Thomas, "LTE-advanced: Next-generation wireless broadband technology", IEEE Wireless Commun. Mag., vol. 17, no. 3, pp. 10-22, Jun. 2010.

[25] G. Wunder et al., "5GNOW: Non-orthogonal, asynchronous waveforms for future mobile applications", IEEE Commun. Mag., pp. 97-105, Feb. 2014.

[26] P. Hansen, N. Mladenovic, and J. A. M. Perez, "Variable neighbourhood search: methods and applications", Annals of Operations Research, vol. 1, pp. 367-407, Mar 2010.

[27] M. H. Hayes, Statistical Digital Signal Processing and Modeling, John Wiley and Sons Ltd, UK, 1996.

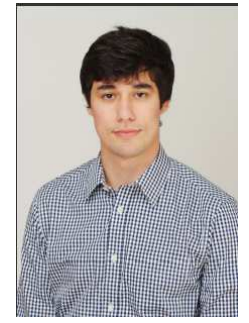

Abderraouf Yamani (born in Algiers, Algeria in 1991) received the B.Eng. degree in electronic engineering and the M.Sc. degree in mobile communications from University of Surrey, U.K., in 2013 and 2014 respectively. He was awarded the 5GIC performance scholarship and is currently pursuing the Ph.D. degree in electronic engineering with Institute for Communication Systems, University of Surrey. His primary research interests include signal processing for communications, with an emphasis on large-scale MIMO precoding and detection.

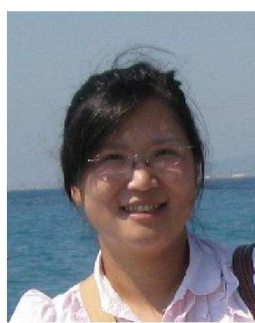

Na Yi (M'10) received the Ph.D. degree in Wireless Communications from the University of Surrey, UK in 2009. Her research interests and expertise cover cognitive radios/networks, advanced signal processing in wireless communications and sensor networks, cooperation and relaying in wireless networks, cognitive resource allocation for wireless networks. She was awarded $\mathrm{PhD}$ studentship from EPSRC, UK in 2005 and strongly involved into the UK Mobile VCE Core 4 Efficiency program on the Cooperation and Cognition for Future Wireles Communications tasks. She led the system structure in C2Power on the cooperative relaying for power saving task, and ACROPOLIS on spectrum awareness and decision execution tasks. She also strongly involved in WHERE on the Location-Aided Cognitive Radio task, and led Location-Aided fixed relays for cellular systems task in WHERE2. Currently, she is focusing on scalable transceiver design for multi-cell in 5GIC and low-complexity relaying design with partial destroyed network infrastructure in RESCUE.

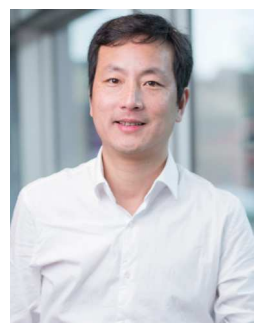

Yi Ma (M'04-SM'10) received the Ph.D. degree in electrical engineering and electronics from The University of Liverpool, U.K., in 2004. He joined Institute for Communication Systems (ICS), University of Surrey, Guildford, U.K., in 2004, where he is currently a member of the academic staff. $\mathrm{He}$ is leading a research group within the ICS to conduct the fundamental research of wireless communication systems covering signal processing and applied information theory. He is also the Chair of Air-Interface Club within the ICS, and the Work Area Leader of $5 \mathrm{G}$ Innovation Centre. $\mathrm{He}$ is the author or co-author of more than 100 peer-reviewed IEEE journal and conference papers in the areas of cooperative communications, cognitive radios, interference utilization, cooperative localization, radio resource allocation, MIMO, estimation, synchronization, modulation and detection techniques. He has 3 patents in the areas of spectrum sensing, signal modulation and detection. He is currently the technical coordinator of FP7 ICT-RESCUE project. Dr. Ma served as the Tutorial Chair for EuroWireless2013, PIMRC2014, CAMAD2015, and he was the founder of Crowd-Net Workshop in conjunction with ICC'15 and ICC'16.

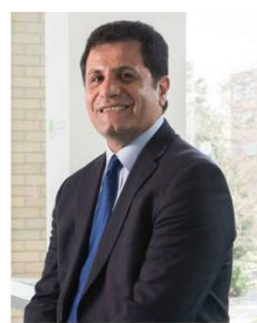

Rahim Tafazolli (SM'09) is a professor and the Director of the Institute for Communication Systems (ICS) and 5G Innovation Centre (5GIC), The University of Surrey in the UK. He has published more than 500 research papers in refereed journals, international conferences and as invited speaker. He is the editor of two books on Technologies for Wireless Future published by Wileys Vol.1 in 2004 and Vol.2 2006. He was appointed as Fellow of WWRF (Wireless World Research Forum) in April 2011, in recognition of his personal contribution to the wireless world. As well as heading one of Europes leading research groups. 